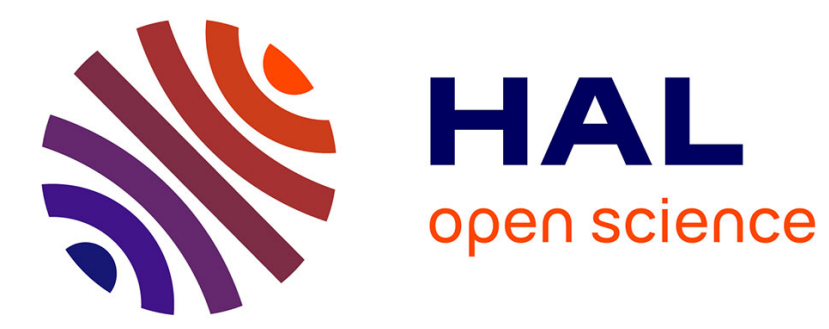

\title{
Robust sensor placement for signal extraction
}

Fateme Ghayem, Bertrand Rivet, Rodrigo Cabral Farias, Christian Jutten

\section{To cite this version:}

Fateme Ghayem, Bertrand Rivet, Rodrigo Cabral Farias, Christian Jutten. Robust sensor placement for signal extraction. IEEE Transactions on Signal Processing, 2021, 69, pp.4513-4528. 10.1109/TSP.2021.3099954 . hal-03325710v3

\section{HAL Id: hal-03325710 \\ https://hal.univ-grenoble-alpes.fr/hal-03325710v3}

Submitted on 25 Aug 2021

HAL is a multi-disciplinary open access archive for the deposit and dissemination of scientific research documents, whether they are published or not. The documents may come from teaching and research institutions in France or abroad, or from public or private research centers.
L'archive ouverte pluridisciplinaire HAL, est destinée au dépôt et à la diffusion de documents scientifiques de niveau recherche, publiés ou non, émanant des établissements d'enseignement et de recherche français ou étrangers, des laboratoires publics ou privés. 


\title{
Robust sensor placement for signal extraction
}

\author{
Fateme Ghayem, Student Member, IEEE, Bertrand Rivet, Rodrigo Cabral Farias, \\ and Christian Jutten, Fellow, IEEE
}

\begin{abstract}
This paper proposes an efficient algorithm for robust sensor placement with the purpose of recovering a source signal from noisy measurements. To model uncertainty on the spatiallyvariant sensors gain and on the spatially correlated noise, we assume that both are realizations of Gaussian processes. Since the signal to noise ratio (SNR) is also uncertain in this context, to achieve a robust signal extraction, we propose a new placement criterion based on the maximization of the probability that the SNR exceeds a given threshold. This criterion can be easily evaluated using the Gaussian process assumption. Moreover, to reduce the computational complexity of the joint maximization of the criterion with respect to all sensor positions, we suggest a sequential maximization approach, where the sensor positions are chosen one at a time. Finally, we present numerical results showing the superior robustness of the proposed approach when compared to standard sensor placement criteria aimed at interpolating the spatial gain and to a recently proposed criterion aimed at maximizing the average SNR.
\end{abstract}

Index Terms-sensor placement, source extraction, signal to noise ratio, Gaussian processes.

\section{INTRODUCTION}

$\mathbf{M}$ ANY signal processing problems can be cast from a generic setting where a source signal propagates through a given structure to the sensors. Under this setting, we can be interested either in (i) characterizing the source signal, or (ii) the structure, or even (iii) the resulting field of signals in some regions of the structure. In all these cases, signals are recorded by multiple sensors located at different positions within or on the structure. Due to price, energy or ergonomic constraints (e.g. when the structure is the human body), the number of sensors is often limited and it becomes crucial to place a few sensors at positions which contain the maximum information. This problem corresponds to optimal sensor placement and it is faced in a great number of applications ranging from infrastructure monitoring [1]-[4] and robotics [5] to biomedical signal processing [6], [7].

The way to tackle the problem of optimal sensor placement differs from one application to another, it mainly depends on which of three aspects mentioned above we want to focus on. In this paper, we study this problem aiming at the first aspect, that is, to extract a source signal from a set of noisy measurements collected from a limited number of candidate sensors. It is important to distinguish between sensor selection

F. Ghayem, B. Rivet and C. Jutten are with the GIPSA-Lab, Department of Images and Signals, University of Grenoble Alpes, France. C. Jutten is also with Institut Universitaire de France, Paris. (email: fateme.ghayem@gmail.com; bertrand.rivet@gipsa-lab.grenoble-inp.fr; christian.jutten@gipsa-lab.grenoble-inp.fr).

R. Cabral Farias is with Univ. Côte d'Azur, CNRS, I3S, 06900 SophiaAntipolis, France (e-mail: cabral@i3s.unice.fr).

This work has been supported by the Center for International Scientific Studies and Collaboration (CISSC), and the European project ERC- 2012AdG320684-CHESS and sensor placement problems. In sensor selection problems, for example those faced in [8]-[10], we have access to the recorded signals coming from all sensors located in the space, and the purpose is then to choose a subset of sensors from all sensors in the space by exploiting the recorded signals. In contrast, in sensor placement problem, we consider a limited number of sensors to be placed in the space, and we do not have the signals coming from these sensors to take the decision. Therefore, the problem dealt with in this paper is fundamentally different from the sensor selection problem.

In what follows we describe the model we consider for the recorded signals. All signals are supposed to be real valued. Scalars are denoted by lower case letters $z$, vectors by lower case boldface letters $\mathbf{z}$ and matrices by upper case boldface letters $\mathbf{Z}$. To retrieve the source of interest $s(t)$ at time $t$, a noisy measurement $y(\mathbf{x}, t)$ is recorded by a sensor positioned at $\mathrm{x} \in \mathcal{X} \subseteq \mathbb{R}^{D}$. The model for representing the sensor measurement mainly depends on what kind of signals we are dealing with. For electrical signals such as electrocardiogram (ECG) and electroencephalography (EEG), the quasi-static approximation of the Maxwell law is satisfied, and we can neglect propagation delays (fast enough propagation) between the source and the sensors. In this case, the following timeinvariant linear instantaneous model is used to represent the noisy measurement:

$$
y(\mathbf{x}, t)=a(\mathbf{x}) s(t)+n(\mathbf{x}, t),
$$

where $a(\mathbf{x})$ is the gain between the source $s(t)$ and the sensor at position $\mathbf{x}$, and $n(\mathbf{x}, t)$ is the corresponding spatially correlated/time uncorrelated additive noise at time $t$.

We note that, in case of using acoustic signals such as phonocardiogram (PCG), we may need to take into account the propagation delay between the source and the sensor, and the measurement modeling should be able to represent the filtering between the sensor and the source. In this case, a linear convolutive mixture model can be used. This type of signals is out of the scope of this paper.

Now, if $M$ sensors are used at locations $\mathbf{X}_{M}=\left\{\mathbf{x}_{1}, \mathbf{x}_{2}, \ldots, \mathbf{x}_{M}\right\}$, then the set of noisy measurements related to each sensor can be denoted by the vector $\mathbf{y}\left(\mathbf{X}_{M}, t\right)=\left[y\left(\mathbf{x}_{1}, t\right), y\left(\mathbf{x}_{2}, t\right), \ldots, y\left(\mathbf{x}_{M}, t\right)\right]^{T}$, where.$T$ is the transpose operator. We also denote $\mathbf{n}\left(\mathbf{X}_{M}, t\right)=\left[n\left(\mathbf{x}_{1}, t\right), n\left(\mathbf{x}_{2}, t\right), \ldots, n\left(\mathbf{x}_{M}, t\right)\right]^{T} \quad$ and $\mathbf{a}\left(\mathbf{X}_{M}\right)=\left[a\left(\mathbf{x}_{1}\right), a\left(\mathbf{x}_{2}\right), \ldots, a\left(\mathbf{x}_{M}\right)\right]^{T}$ the corresponding noise and spatial gain vectors, respectively. One can then write the measurement model (1) in a more compact form

$$
\mathbf{y}\left(\mathbf{X}_{M}, t\right)=\mathbf{a}\left(\mathbf{X}_{M}\right) s(t)+\mathbf{n}\left(\mathbf{X}_{M}, t\right) .
$$

The main goal is to find the optimal set of locations $\mathbf{X}_{M}$, such that the best estimation of the source $s(t)$ can be obtained from 
the measurements $\mathbf{y}\left(\mathbf{X}_{M}, t\right)$. We further assume that $a(\mathbf{x})$ is not fully known, either because we have measured it only in a few positions, or because the available prior information on it is vague. To model this uncertainty, we take a Bayesian perspective and we assume that $a(\mathbf{x})$ is a realization of a Gaussian process (GP). Note that, there are several reasons that a GP is a suitable choice to model the spatial gain. A GP is a very flexible signal model, since it does not directly model the signal shape, but the second order statistical properties. Thus, it is able to model signal with very various shapes, by only controlling a few parameters. Due to the hyper-parameters of the GP, we can control different characteristics of $a(\mathbf{x})$. For example, we can control its smoothness or its magnitude. Also, even for fixed values of the parameters, we can represent different signal shapes. And finally, thanks to the properties the GP obtains from the normal distribution, the computation of various quantities such as marginal and conditional distributions is straightforward [11]. Such uncertainty modeling approach has been extensively used in function interpolation, where it is named kriging [12], [13], in the design of computer experiments [14] and in global optimization [15]. It has also been used for sensor placement in [16], where the increment of the mutual information (MI) is proposed as a placement criterion. In that work, the increment in MI is maximized using a near-optimal greedy approach. New sensors are added one by one starting with an empty set, in such a way that, at each iteration, the selected location adds maximum information.

The approach in [16] focuses on interpolating a field of signals e.g. room temperature. As any other kriging based criterion for interpolation [17], [18], this approach could be used in our setting if the goal was to estimate $a(\mathbf{x})$. But as previously stated, this is not our aim, and the two goals may not be equivalent. Consider a simple example where $a(\mathbf{x})$ has similar uncertainty everywhere in $\mathcal{X}$ and that $n(\mathbf{x}, t)$ has a short correlation length. In this case, interpolation-based criteria will spread the sensors over $\mathcal{X}$, while a criterion targeting the extraction of $s(t)$ will spread them across positions with higher $|a(\mathbf{x})|$.

The goal we pursue in this paper is similar to what has been recently studied in [19]. In that paper, by considering a GP model for $a(\mathbf{x})$ and $n(\mathbf{x}, t)$ and using a linear source extraction approach, the output signal-to-noise-ratio (SNR) of the estimated source signal is derived as a function of $a(\mathbf{x})$ and of the sensor locations $\mathbf{X}_{M}$. Since $a(\mathbf{x})$ is stochastic, the SNR is also stochastic. In [19] the average SNR is suggested as the criterion to solve the problem of optimal sensor placement for source extraction. This suggested criterion significantly outperforms kriging-based methods in terms of the SNR. However, this method does not provide an analytic characterization for the regions where placing new sensors degrades the extraction of the source due to the uncertainty of the spatial gain. In this paper, we go beyond the idea of maximizing the average SNR, and we present a new criterion based on the probability density function (pdf) of the SNR. To this end, we first derive the pdf of the SNR. Then, by having the distribution of the SNR, we suggest to maximize the probability of the SNR to be greater than a threshold, and we will show that the new probabilistic criterion is more robust against the uncertainty of the spatial gain.

In this paper, we propose to extend the idea in [19] to take into account the robustness issue presented above. A more general criterion, based on the probability that the SNR exceeds a fixed threshold is put forward. Since the evaluation of this criterion requires the pdf of the SNR, we indicate how this pdf can be evaluated using the GP assumption on the spatial gain. Moreover, when we choose the sensor positions in a sequential fashion, we show that the criterion can then be evaluated analytically. Simulation results obtained with the sequential approach show that the threshold in the probabilitybased criterion can be adjusted to a trade-off between having a maximum increase in the SNR and being robust to a possible decrease in the SNR due to uncertainty. Also, the results confirm the superior robustness of our approach compared to other criteria from the literature.

The rest of the paper is organized as follows. In Section II we derive the criterion based on the SNR and we show how to evaluate the required pdf. Section III presents how the criterion becomes simpler with the sequential approach and in Section IV, we briefly describe two kriging-based existing criteria. In Section V, the simulation results are presented. Finally, section VI concludes the paper and discusses perspectives.

\section{OPtimAL SENSOR PLACEMENT FOR SOURCE EXTRACTION}

In this section, we are going to solve the problem of optimal sensor placement for source extraction using the noisy sensor measurements model given by (2). We first provide a brief review of the source extraction problem. Then, we present the proposed criterion.

\section{A. Linear signal extraction and signal to noise ratio}

Let us consider the observation model in (2). By using a linear estimator described $\mathbf{f} \in \mathbb{R}^{M}$, the extracted source signal $\hat{s}(t)$ is the following [19]:

$$
\hat{s}(t)=\mathbf{f}^{T} \mathbf{y}\left(\mathbf{X}_{M}, t\right)=\mathbf{f}^{T} \mathbf{a}\left(\mathbf{X}_{M}\right) s(t)+\mathbf{f}^{T} \mathbf{n}\left(\mathbf{X}_{M}, t\right) .
$$

For simplicity, in the rest of this paper, the dependence on the set of sensor locations $\mathbf{X}_{M}$ will be denoted with a subscript $(\cdot)_{M}$. For example, a function $\mathbf{P}\left(\mathbf{X}_{M}\right)$ will be denoted $\mathbf{P}_{M}$. From (3), the SNR of the estimation of $s(t)$ can be derived as

$$
\operatorname{SNR}\left(\mathbf{f} \mid \mathbf{X}_{M}\right)=\frac{\mathbb{E}_{t}\left[\left(\mathbf{f}^{T} \mathbf{a}_{M} s(t)\right)^{2}\right]}{\mathbb{E}_{t}\left[\left(\mathbf{f}^{T} \mathbf{n}_{M}(t)\right)^{2}\right]}=\frac{\sigma_{s}^{2} \mathbf{f}^{T} \mathbf{a}_{M} \mathbf{a}_{M}^{T} \mathbf{f}}{\mathbf{f}^{T} \mathbf{C}_{M M}^{n} \mathbf{f}},
$$

where $\mathbb{E}_{t}[\cdot]$ stands for the expectation over time, and the signal time samples are temporally zero-mean, independent and identically distributed (iid) with $\sigma_{s}^{2}=\mathbb{E}\left[s(t)^{2}\right]$ and $\mathbf{C}_{M M}^{n}=\mathbb{E}\left[\mathbf{n}_{M}(t) \mathbf{n}_{M}^{T}(t)\right]$. From (4), it can be seen that the SNR is a function of both the extraction vector $\mathbf{f}$ and sensor locations $\mathbf{X}_{M}$. By considering the SNR as a function of $\mathbf{f}$ and maximizing the output SNR over $\mathbf{f}$, assuming that the sensor positions $\mathbf{X}_{M}$ are fixed and known, the best linear estimator $\mathbf{f}^{*}$ classically is given by

$$
\mathbf{f}^{*} \mid \mathbf{X}_{M}=\mathbf{R}_{M M} \mathbf{a}_{M},
$$


where $\mathbf{R}_{M M} \triangleq\left(\mathbf{C}_{M M}^{n}\right)^{-1}$. By conditioning on $\mathbf{X}_{M}$ we note that the maximization over $\mathbf{f}$ is done under the assumption that the sensor positions $\mathbf{X}_{M}$ are known. For simplicity, in the rest of this paper, we denote the best extractor just by $\mathbf{f}^{*}$. By replacing (5) in (4), the corresponding SNR is

$$
\operatorname{SNR}\left(\mathbf{f}^{*} \mid \mathbf{X}_{M}\right)=\sigma_{s}^{2} \mathbf{a}_{M}^{T} \mathbf{R}_{M M} \mathbf{a}_{M} .
$$

Now, by observing the resulting output SNR in (6) as a function of sensor locations $\mathbf{X}_{M}$ and maximizing it over $\mathbf{X}_{M}$, we end up with the optimal solution for sensor placement in terms of the best SNR. This solution implicitly assumes a perfect knowledge of spatial gains $a\left(\mathbf{x}_{i}\right), i \in\{1,2, \ldots, M\}$. However, such a perfect knowledge is not always available. To overcome this issue a GP with mean $m^{a}(\mathbf{x})$ and covariance function $C^{a}\left(\mathbf{x}, \mathbf{x}^{\prime}\right)$ is considered to model $a(\mathbf{x})$ as $\hat{a}(\mathbf{x})$ :

$$
\hat{a}(\mathbf{x}) \sim \mathcal{G P}\left(m^{a}(\mathbf{x}), C^{a}\left(\mathbf{x}, \mathbf{x}^{\prime}\right)\right) .
$$

Consequently, the randomness of $a(\mathbf{x})$ leads to a stochastic SNR as well, which is estimated as:

$$
\widehat{\operatorname{SNR}}\left(\hat{\mathbf{f}} \mid \mathbf{X}_{M}\right)=\sigma_{s}^{2} \hat{\mathbf{a}}_{M}^{T} \mathbf{R}_{M M} \hat{\mathbf{a}}_{M},
$$

where $\hat{\mathbf{f}}=\mathbf{R}_{M M} \hat{\mathbf{a}}_{M}$.

Remark 1. If we have a prior information on $\mathbf{a}_{K}=$ $\left[a\left(\mathbf{x}_{1}\right), a\left(\mathbf{x}_{2}\right), \ldots, a\left(\mathbf{x}_{K}\right)\right]^{T}$, for example if we have previously placed the sensors at these positions and measured the gains, then the prior (7) can be updated to include this information, simply by conditioning the GP on the measurements of the gains. The conditioned GP is still a GP but with conditioned mean and covariance functions [11].

To target the SNR in (6), we need to use a quantitative statistical measure of the SNR distribution which is robust against the uncertainty in the spatial gain model (7). This will be presented in the next part.

\section{B. Proposed criterion}

Since the pdf of a random variable contains all the information about it, here, by having the pdf of the SNR, we are able to define different efficient statistical criteria. Therefore, first, we attempt to derive the pdf of the SNR given in (8). Then, based on the obtained pdf, we present our robust criterion for sensor placement.

To simplify notation, we define the random variable $W_{M} \triangleq$ $\left(1 / \sigma_{s}^{2}\right) \operatorname{SNR}\left(\mathbf{f}^{*} \mid \mathbf{X}_{M}\right)=\mathbf{a}_{M}^{T} \mathbf{R}_{M M}^{n} \mathbf{a}_{M}$. Now, in order to find the distribution of $W_{M}$, we have the following proposition:

Lemma 1. (Distribution of $W_{M}$ ) If the spatial gain $a(\mathbf{x})$ follows the GP model $a(\mathbf{x}) \sim \mathcal{G P}\left(m^{a}(\mathbf{x}), C^{a}\left(\mathbf{x}, \mathbf{x}^{\prime}\right)\right)$, for any positive integer number $M$, then $W_{M}$ is the weighted sum of $M$ independent random variables as follows:

$$
W_{M}=\sum_{i=1}^{M} d_{i} y_{i}^{2},
$$

where $y_{i}$ are independent normally distributed random variables $y_{i} \sim \mathcal{N}\left(m_{y_{i}}, 1\right)$, with $m_{y_{i}}=\mathbf{u}_{i}^{T}\left(\boldsymbol{C}_{M M}^{a}\right)^{-\frac{1}{2}} \mathbf{m}_{M}^{a}$, and, $d_{i}$ and $\mathbf{u}_{i}$ are respectively the eigenvalues and eigenvectors of the matrix $\boldsymbol{A}$ defined as follows:

$$
\boldsymbol{A} \triangleq\left(\boldsymbol{C}_{M M}^{a}\right)^{\frac{1}{2}} \boldsymbol{R}_{M M}\left(\boldsymbol{C}_{M M}^{a}\right)^{\frac{1}{2}},
$$

and $\mathbf{m}_{M}^{a}=\left[m^{a}\left(\mathbf{x}_{1}\right), m^{a}\left(\mathbf{x}_{2}\right), \ldots, m^{a}\left(\mathbf{x}_{M}\right)\right]$.

Proof: See Appendix A.

Remark 2. Considering (46), it is straightforward to show that for any $i \neq j \in\{1,2, \ldots, M\}, y_{i}$ and $y_{j}$ are uncorrelated. On the other hand, $y_{i}$ and $y_{j}$ are jointly normally distributed. Therefore, $y_{i}$ and $y_{j}$ are independent. Moreover, as long as the Jacobian of a non-linear transformation $h: \mathbb{R}^{M} \longrightarrow \mathbb{R}^{M^{\prime}}$ is diagonal, the non-linear functions will not affect the independence of the random variables. Therefore, by defining $h\left(y_{i}\right) \triangleq d_{i} y_{i}^{2}$, since the Jacobian matrix of the function $h$ over the independent random variables $y_{i}, i \in\{1,2, \ldots, M\}$ is diagonal, it can be concluded that for any $i \neq j \in\{1,2, \ldots, M\}, \gamma_{i} \triangleq d_{i} y_{i}^{2}$ and $\gamma_{j} \triangleq d_{j} y_{j}^{2}$ are also independent.

Remark 3. Since $y_{i}$ is a normally distributed scalar with non-zero mean $m_{y_{i}}$ and variance one, its squared form $y_{i}^{2}$ follows a noncentral chi-squared distribution with the number of degrees of freedom $k_{i}=1$, and non-centrality parameter $\lambda_{i}=m_{y_{i}}^{2}$. So, the pdf of the random variable $v_{i} \triangleq y_{i}^{2}$ becomes $g_{V_{i}}\left(v_{i} ; k_{i}, \lambda_{i}\right)=\frac{1}{2} \exp ^{-\frac{\left(v_{i}+\lambda_{i}\right)}{2}}\left(\frac{v_{i}}{\lambda_{i}}\right)^{\left(\frac{k_{i}}{4}-\frac{1}{2}\right)} I_{\frac{k_{i}}{2}-1}\left(\sqrt{\lambda_{i} v_{i}}\right)$,

where I.(.) is the modified Bessel function of the first kind. Now, by defining $\gamma_{i}=d_{i} v_{i}$ to be the scaled form of the random variable $v_{i}$ with the positive scale factor $d_{i}$, the distribution of $\gamma_{i}$ becomes as follows:

$$
g_{\Gamma_{i}}\left(\gamma_{i}\right)=\frac{1}{d_{i}} g_{V_{i}}\left(\frac{\gamma_{i}}{d_{i}} ; k_{i}, \lambda_{i}\right),
$$

denoting $g_{V_{i}}($.$) the pdf of the random variable v_{i}=y_{i}^{2}$ with noncentral chi-squared distribution defined in (11).

From Remark 2 and Remark 3, it can be concluded that the SNR is the sum of $M$ independent random variables $\gamma_{i}$ each having a pdf defined in (12). Therefore, due to independence, the pdf of the SNR is given by the convolution product, denoted by $*$, between the pdf of $M$ random variables $\gamma_{i}$ :

$$
\begin{array}{r}
g_{W_{M}}\left(w_{M}\right)=g_{\Gamma_{1}}\left(w_{M}\right) * g_{\Gamma_{2}}\left(w_{M}\right) * \cdots * g_{\Gamma_{M}}\left(w_{M}\right) \\
=\frac{1}{\prod_{i=1}^{M} d_{i}} g_{V_{1}}\left(\frac{w_{M}}{d_{1}}\right) * g_{V_{2}}\left(\frac{w_{M}}{d_{2}}\right) * \cdots * g_{V_{M}}\left(\frac{w_{M}}{d_{M}}\right) .
\end{array}
$$

Note that, for simplicity, in the above, the parameters $k_{i}$ and $\lambda_{i}$ are omitted, and we presented $g_{V_{i}}\left(v_{i} ; k_{i}, \lambda_{i}\right)$ by $g_{V_{i}}\left(v_{i}\right)$.

Now, by knowing the pdf of the SNR, it is possible to define different criteria. To have an efficient criterion, it is necessary to consider two important properties: first, the criterion has to suggest positions providing maximum output SNR. Second, the criterion should be robust against the uncertainty on the gains, that is, it should avoid positions that have a nonnegligible probability of generating a small SNR. So, to achieve these two goals, we propose to search for a set of 

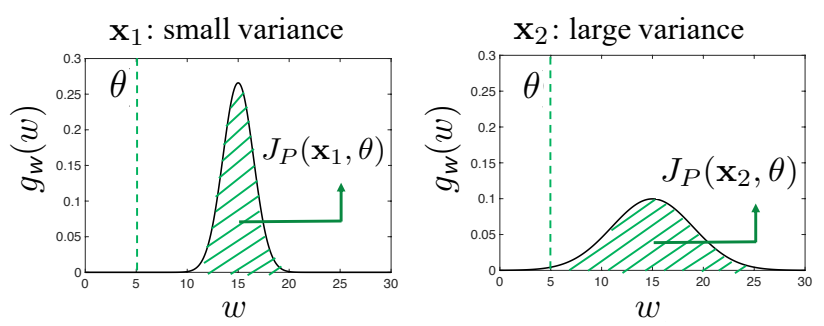

(a) Case 1: Small $\theta$
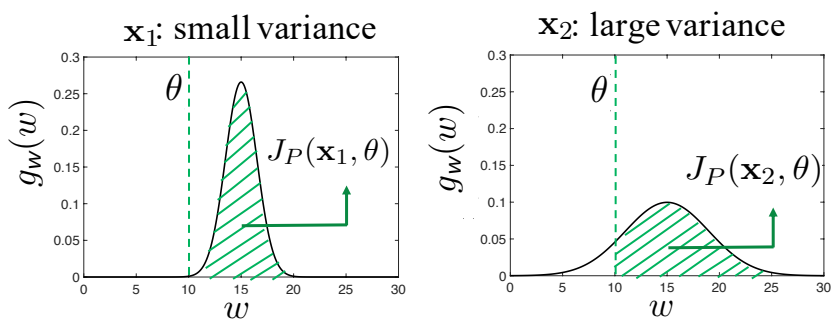

(b) Case 2: Large $\theta$

Fig. 1: In this figure the influence of the parameter $\theta$ on the proposed criterion $J_{P}$ is demonstrated. To this end, we consider two positions $\mathbf{x}_{1}$ with small uncertainty, and $\mathbf{x}_{2}$ with large uncertainty on the spatial gain. The pdf of the random variable $W$ is plotted, and the green shadows represent $J_{P}$ for a given parameter $\theta$. In the first case, we choose a small value for the parameter $\theta$, and in the second case, this parameter is increased.

positions that maximizes the probability of the SNR to be greater than a threshold denoted $\theta$. This leads to the following problem:

$$
\hat{\mathbf{X}}_{M}=\underset{\mathbf{X}_{M}}{\operatorname{argmax}} J_{P}\left(\mathbf{X}_{M}, \theta\right),
$$

where the criterion $J_{P}\left(\mathbf{X}_{M}, \theta\right)$ is based on the cumulative distribution function (cdf) of the SNR:

$$
J_{P}\left(\mathbf{X}_{M}, \theta\right)=\operatorname{Pr}\left(W_{M} \geqslant \theta\right)=1-G_{W_{M}}(\theta),
$$

where $G_{W_{M}}(\cdot)$ represents the cdf of $S N R / \sigma_{s}^{2}$ (i.e. $W_{M}$ ) conditioned to the sensors at positions $\mathbf{X}_{M}$. By knowing the pdf of $W_{M}$, from (13) the cdf becomes as follows:

$$
\begin{gathered}
G_{W_{M}}(\theta ; \mathbf{k}, \Lambda)=\frac{1}{\prod_{i=1}^{M} d_{i}} \times \\
G_{V_{1}}\left(\frac{\theta}{d_{1}} ; k_{1}, \lambda_{1}\right) * g_{V_{2}}\left(\frac{\theta}{d_{2}} ; k_{2}, \lambda_{2}\right) * \cdots * g_{V_{M}}\left(\frac{\theta}{d_{M}} ; k_{M}, \lambda_{M}\right),
\end{gathered}
$$

where $\mathbf{k}=\left\{k_{1}, k_{2}, \ldots, k_{M}\right\}, \Lambda=\left\{\lambda_{1}, \lambda_{2}, \ldots, \lambda_{M}\right\}$, and $G_{V_{1}}(\cdot)$ is the corresponding cdf of $g_{V_{1}}(\cdot)$. Note that from Remark 3 we also have:

$$
G_{V_{1}}\left(v_{1} ; k_{1}, \lambda_{1}\right)=1-Q_{\frac{k_{1}}{2}}\left(\sqrt{\lambda_{1}}, \sqrt{v_{1}}\right),
$$

where $\lambda_{i}$ and $k_{i}$ are defined in (11), and $Q$ is the Marcum-Qfunction [20].

Remark 4. (Relation between $J_{E}$ and $J_{P}$ ) Taking into account that the random variable $W_{M}$ is non-negative, and using the Markov's inequality, we can have the following link between the proposed criterion $J_{P}=\operatorname{Pr}\left(W_{M} \geqslant \theta\right)$ and the average $S N R J_{E}=\sigma_{s}^{2} \cdot \mathbb{E}\left[W_{M}\right]$ :

$$
J_{E} \geq \sigma_{s}^{2} \cdot \theta \cdot J_{P}
$$

Therefore, for a given $\theta$, by maximizing $J_{P}$, simultaneously, we implicitly maximize the average SNR. Note that, although by maximizing $J_{P}$ we maximize a lower bound of the average SNR, we still cannot guarantee that the average SNR is maximized, and so, by reducing the risk of choosing a position with high level of uncertainty of the spatial gain, we have a sacrifice which is the fact that the selected position does not necessarily have the maximum average SNR.
Remark 5. (Links with maximum likelihood) It is worth mentioning that if we consider a target value $\alpha$ of $S N R / \sigma_{s}^{2}$, then the maximum likelihood can also be used as a sensor placement criterion. In this case, we look for the set $\boldsymbol{X}_{M}$ maximizing $J_{p}\left(\mathbf{X}_{M}, \alpha\right)=g_{W_{M}}(\alpha)$. However, this criterion does not have an intuitive interpretation regarding robustness and maximization of SNR as (15).

Remark 6. (Influence of parameter $\theta$ on $J_{P}$ ) Parameter $\theta$ in the proposed criterion represents how much the criterion discriminates between the positions with different levels of uncertainty on the spatial gain. Fig. 1 is provided to show the influence of the parameter $\theta$ in the behavior of $J_{P}$. In this figure, we have assumed a small uncertainty on the spatial gain at position $\mathbf{x}_{1}$, and a large uncertainty at position $\mathbf{x}_{2}$. Then, we have plotted the pdf of the SNR, and for two different values of $\theta$, the proposed criterion is depicted in green shadows. Comparing Fig. 1-a and Fig. 1-b, we can see that by increasing the parameter $\theta$, the proposed criterion is more discriminative, and it takes larger values at positions where we have smaller uncertainty on the spatial gain. We note that this interpretation is valid for $\theta$ less than the median of the pdf of the SNR.

The advantage of criterion (15) is that the free parameter $\theta$ can be used to control the risk of locating a new sensor at position with high level of uncertainty of the spatial gain. In other words, by increasing $\theta$ to sufficiently high values, the upper tail of the output SNR will be compared for different positions, forcing the criterion to be high for positions leading to a high SNR but which are conservative. In this case, the positions leading to very high average SNR but with large dispersion will be discarded.

On the contrary, by reducing the parameter $\theta$ to a sufficiently low value, most of the positions will lead to similar criterion values, and, as a consequence, sensor positions where we have a high uncertainty on the spatial gain (e.g. $\mathrm{x}_{2}$ in Fig. 1), and at the same time, lead to a very high average SNR values, will not be discarded. So, unlike maximum likelihood, with this criterion, depending on the application, we can make a tradeoff between achieving a sufficiently high SNR and reducing the risk that the true output SNR will be much lower than expected. 


\section{Sequential SEnSOR PLACEMENT}

In this section, we first discuss about the computational complexity of the sensor placement problem for $M$ sensors. Two solutions that reduce the complexity are then presented. One solution is based on a greedy method for breaking the underlying maximization problem into smaller problems. The second solution follows the same greedy approach, but it exploits information on the spatial gain that may be available when the smaller sensor placement problems are solved sequentially.

\section{A. Computational cost}

In practice, to optimize the criterion (15), a grid search is used. We are thus looking for the best subset $\mathbf{X}_{M}$ from a total spatial grid of $T$ positions $\mathbf{X}_{T}$ to place $M$ sensors such that our proposed criterion in (15) is maximized. The optimal solution to this problem requires a combinatorial search over $\frac{T !}{M !(T-M) !}$ possibilities. This search has a high computational cost, especially if the grid is very tight, i.e. when $T$ is large. Therefore, it is essential to provide a less costly solution. In the following, we present a straightforward way to tackle this problem.

\section{B. Greedy method}

One approach to reduce the computational complexity of the combinatorial search is to use a greedy method. In this approach, we start from an empty set and iteratively add $N<M$ sensors such that at each selection, the underlying criterion becomes maximum for the totally selected positions up to each iteration. Assume that $K$ sensors have already been located at $\mathbf{X}_{K}$ and we want to add the next $N$ new sensors at $\mathbf{X}_{N}$ where $N$ is smaller than the total number of sensors $M$. Among all the possibilities for the $N$ new sensors, we search for the subset $\mathbf{X}_{N}$ such that $\mathbf{X}_{K+N}=\left\{\mathbf{X}_{K} \cup \mathbf{X}_{N}\right\}$ maximizes (15). Since at each iteration $N<M$, the computational complexity will be reduced compared with a direct search for $\mathbf{X}_{M}$. However, such complexity reduction comes at the expense of a possible suboptimality of the chosen sensor positions.

\section{Sequential approach}

When breaking the larger search for the $M$ optimal positions into a sequential search for $N$ positions, we may be interested in using the extraction system with the new sensors, prior to choosing and placing the next $N$ sensors. In such a case, it is possible to use the available measurements not only to extract the signal but also to obtain new information on the spatial gains. If such information can be retrieved, then it can be used to reduce the uncertainty on $\hat{a}(\mathbf{x})$ and, as a consequence, to reduce the uncertainty on the SNR for the next $N$ sensors to be placed. Note that this sequential approach is different from the greedy method, whose only objective is to iteratively maximize the criterion and not to retrieve any other useful information that may reduce the uncertainty.

In practice, information on the spatial gains may be obtained either if a ground truth signal is available [7], or with blind

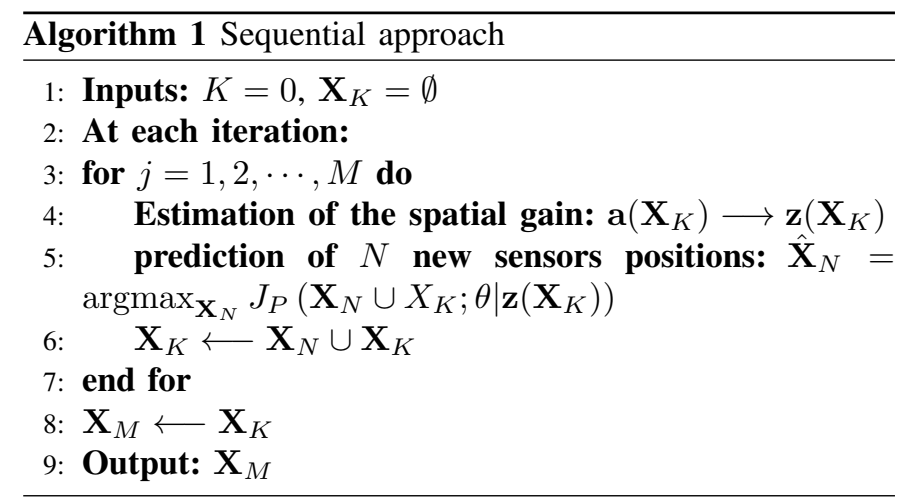

signal processing techniques such as independent component analysis (ICA) [21] or sparse component analysis (SCA) [22]. In the following, we suppose that one of these approaches has been used and that the new information available on the gain is equivalent to noisy measurements of it, denoted $\mathbf{z}_{K}$, obtained at the previously placed sensor positions $\mathbf{X}_{K}$ :

$$
\mathbf{z}_{K}=\mathbf{a}_{K}+\mathbf{v}_{K}
$$

where $\mathbf{v}_{K}(\mathbf{x})$ is a Gaussian process independent of $\mathbf{a}(\mathbf{x})$ and $\mathbf{n}(\mathbf{x}, t)$, and with the covariance function $C^{v}\left(\mathbf{x}, \mathbf{x}^{\prime}\right)$.

Once we placed the $K$ sensors and measured $\mathbf{z}_{K}$, we can update the criterion for placing the new $N$ sensors by conditioning the gain on $\mathbf{z}_{K}$. The gain vector can then be written as $\hat{\mathbf{a}}_{M}^{T}=\left[\hat{\mathbf{a}}_{K}^{T} \hat{\mathbf{a}}_{N}^{T}\right]$, where $\hat{\mathbf{a}}_{K}$ are the gains for which we have measurements, $\hat{\mathbf{a}}_{N}$ is the random vector corresponding to the spatial gains at candidate positions $\boldsymbol{X}_{N}$, and both are conditioned on $\mathbf{z}_{K}$. As mentioned in Remark 1, since both the gains and $\mathbf{z}_{K}$ are Gaussian, the conditioned gains are also Gaussian with mean and covariance given by [11]

$$
\begin{aligned}
& \mathbf{m}_{M \mid K}=\mathbb{E}\left[\hat{\mathbf{a}}_{M} \mid \mathbf{z}_{K}\right] \\
& =\mathbf{m}_{M}+C_{M K}^{a}\left[C_{K K}^{a}+C_{K K}^{v}\right]^{-1}\left(\mathbf{z}_{K}-\mathbf{m}_{K}\right),
\end{aligned}
$$

and

$$
\begin{aligned}
\boldsymbol{C}_{M \mid K}^{a} & =\mathbb{E}\left[\left(\hat{\mathbf{a}}_{M}-\mathbf{m}_{M \mid K}\right)\left(\hat{\mathbf{a}}_{M}-\mathbf{m}_{M \mid K}\right)^{T}\right] \\
& =C_{M M}^{a}-\boldsymbol{C}_{M K}^{a}\left[\boldsymbol{C}_{K K}^{a}+\boldsymbol{C}_{K K}^{v}\right]^{-1}\left(\boldsymbol{C}_{M K}^{a}\right)^{T}
\end{aligned}
$$

where $C_{M K}^{a}, C_{K K}^{a}$ are blocks of the partitioned covariance matrix $\boldsymbol{C}_{M M}^{a}=\left[\begin{array}{ll}\boldsymbol{C}_{M K}^{a} & \boldsymbol{C}_{M N}^{a}\end{array}\right]=\left[\begin{array}{cc}\boldsymbol{C}_{K K}^{a} & \boldsymbol{C}_{K N}^{a} \\ \boldsymbol{C}_{K N}^{a}{ }_{T} & \boldsymbol{C}_{N N}^{a}\end{array}\right]$.

The estimated SNR is similar to (8) $\widehat{\operatorname{SNR}}\left(\hat{\mathbf{f}} \mid \mathbf{X}_{N}, \mathbf{z}_{K}\right)$, but, since $\mathbf{X}_{K}$ is fixed, it is now conditioned on $\mathbf{z}_{K}$ and a function of $\mathbf{X}_{N}$ only. As $\hat{\mathbf{a}}_{M}$ is Gaussian, the distribution of $W_{M \mid K} \triangleq$ $\left(1 / \sigma_{s}^{2}\right) \widehat{\operatorname{SNR}}\left(\hat{\mathbf{f}} \mid \mathbf{X}_{N}, \mathbf{z}_{K}\right)$ can be obtained in a similar way as presented in Subsection II-B. With the distribution of $W_{M \mid K}$, we can modify the criterion (15) to have the following robust sequential sensor placement criterion:

$$
J_{P}\left(\mathbf{X}_{N}, \theta \mid \mathbf{z}_{K}\right)=\operatorname{Pr}\left(W_{M \mid K} \geqslant \theta\right)=1-G_{W_{M \mid K}}(\theta) .
$$

The final algorithm of the proposed method using sequential approach is presented in Algorithm 1. 
1) Perfect gain information: if the information on the gain is assumed to be perfect, that is, $\mathbf{z}_{K}=\mathbf{a}_{K}$, then the vector of gains can be written as $\hat{\mathbf{a}}_{M}^{T}=\left[\mathbf{a}_{K}^{T} \hat{\mathbf{a}}_{N}^{T}\right]$, where $\mathbf{a}_{K}$ are now deterministic and $\hat{\mathbf{a}}_{N}$ is a random vector conditioned on the observed $\mathbf{a}_{K}$. To write the conditional SNR, we partition $\boldsymbol{R}_{M M}=\left[\begin{array}{ll}\boldsymbol{R}_{K K} & \boldsymbol{R}_{K N} \\ \boldsymbol{R}_{K N}^{T} & \boldsymbol{R}_{N N}\end{array}\right]$, and then we have:

$$
\begin{gathered}
\frac{1}{\sigma_{s}^{2}} \widehat{\operatorname{SNR}}\left(\hat{\mathbf{f}} \mid \boldsymbol{X}_{M}, \mathbf{a}_{K}\right)=\left[\begin{array}{ll}
\mathbf{a}_{K}^{T} & \hat{\mathbf{a}}_{N}^{T}
\end{array}\right]\left[\begin{array}{cc}
\boldsymbol{R}_{K K} & \boldsymbol{R}_{K N} \\
\boldsymbol{R}_{K N}^{T} & \boldsymbol{R}_{N N}
\end{array}\right]\left[\begin{array}{c}
\mathbf{a}_{K} \\
\hat{\mathbf{a}}_{N}
\end{array}\right] \\
=\mathbf{a}_{K}^{T} \boldsymbol{R}_{K K} \mathbf{a}_{K}+2 \mathbf{a}_{K}^{T} \boldsymbol{R}_{K N} \hat{\mathbf{a}}_{N}+\hat{\mathbf{a}}_{N}^{T} \boldsymbol{R}_{N N} \hat{\mathbf{a}}_{N} \cdot(21)
\end{gathered}
$$

The conditioned random vector $\hat{\mathbf{a}}_{N}$ is also Gaussian, its mean $\mathbf{m}_{N \mid K}$ and covariance $C_{N \mid K}^{a}$ can be obtained respectively from (18) and (19) by setting the noise covariance equal to zero, i.e. $C_{K K}^{v}=\mathbf{0}$. They are given by

$$
\mathbf{m}_{N \mid K}=\mathbb{E}\left[\hat{\mathbf{a}}_{N}\right]=\mathbf{m}_{N}+\left(C_{K N}^{a}\right)^{T}\left(C_{K K}^{a}\right)^{-1}\left(\mathbf{a}_{K}-\mathbf{m}_{K}\right) .
$$

and

$$
\begin{aligned}
\boldsymbol{C}_{N \mid K}^{a} & =\mathbb{E}\left[\left(\hat{\mathbf{a}}_{N}-\mathbf{m}_{N \mid K}\right)\left(\hat{\mathbf{a}}_{N}-\mathbf{m}_{N \mid K}\right)^{T}\right] \\
& =\boldsymbol{C}_{N N}^{a}-\boldsymbol{C}_{N K}^{a}\left(\boldsymbol{C}_{K K}^{a}\right)^{-1}\left(\boldsymbol{C}_{N K}^{a}\right)^{T}
\end{aligned}
$$

Assuming that $\boldsymbol{R}_{N N}$ is invertible and by factorizing (21), we have

$$
\begin{aligned}
& \widehat{\operatorname{SNR}}\left(\hat{\mathbf{f}} \mid \boldsymbol{X}_{N}, \mathbf{a}_{K}\right)=\sigma_{s}^{2} \times \\
& {\left[\left(\hat{\mathbf{a}}_{N}+\boldsymbol{R}_{N N}^{-1} \boldsymbol{R}_{N K} \mathbf{a}_{K}\right)^{T} \boldsymbol{R}_{N N}\left(\hat{\mathbf{a}}_{N}+\boldsymbol{R}_{N N}^{-1} \boldsymbol{R}_{N K} \mathbf{a}_{K}\right)\right.} \\
& \left.\quad+\mathbf{a}_{K}^{T}\left(\boldsymbol{R}_{K K}-\boldsymbol{R}_{K N} \boldsymbol{R}_{N N}^{-1} \boldsymbol{R}_{K N}^{T}\right) \mathbf{a}_{K}\right]
\end{aligned}
$$

Note that the first term is a quadratic form of a Gaussian vector, while the second term is deterministic. Therefore, the random vector

$$
\begin{aligned}
W_{N \mid K} & \triangleq\left(1 / \sigma_{s}^{2}\right) \widehat{\operatorname{SNR}}\left(\hat{\mathbf{f}} \mid \mathbf{X}_{N}, \mathbf{a}_{k}\right) \\
& -\mathbf{a}_{K}^{T}\left(\boldsymbol{R}_{K K}-\boldsymbol{R}_{K N} \boldsymbol{R}_{N N}^{-1} \boldsymbol{R}_{K N}^{T}\right) \mathbf{a}_{K}
\end{aligned}
$$

has a distribution that can be obtained again as described in Subsection II-B. Similarly to (15), a criterion $J_{P}\left(\mathbf{X}_{N}, \theta \mid \mathbf{a}_{K}\right)$ can also be defined.

2) Adding one sensor at a time under the perfect gain information setting: a special case for which $J_{P}\left(\mathbf{X}_{N}, \theta \mid \mathbf{a}_{K}\right)$ has a known analytical form is when we add new sensors one by one, i.e. $N=1$. In this case, $\hat{a}_{N}$ is a scalar random variable and both $R_{N N}$ and $C_{N \mid K}^{a}$ are also scalars. Therefore, we can rewrite (24) as follows

$\widehat{\operatorname{SNR}}\left(\hat{\mathbf{f}} \mid \boldsymbol{X}_{N}, \mathbf{a}_{K}\right)=\sigma_{s}^{2} \times$

$\left[R_{N N}\left(a_{N}+\frac{\boldsymbol{R}_{N K} \mathbf{a}_{K}}{R_{N N}}\right)^{2}+\mathbf{a}_{K}^{T}\left(\boldsymbol{R}_{K K}-\frac{\boldsymbol{R}_{K N} \boldsymbol{R}_{K N}^{T}}{R_{N N}}\right) \mathbf{a}_{K}\right]$

If we define $q_{N} \triangleq\left(a_{N}+\frac{\boldsymbol{R}_{N K} \mathbf{a}_{K}}{R_{N N}}\right) / \sqrt{C_{N \mid K}^{a}}$, then

$$
\begin{aligned}
& \widehat{\operatorname{SNR}}\left(\hat{\mathbf{f}} \mid \boldsymbol{X}_{N}, \mathbf{a}_{K}\right)=\sigma_{s}^{2} \times \\
& {\left[R_{N N} C_{N \mid K}^{a} q_{N}^{2}+\mathbf{a}_{K}^{T}\left(\boldsymbol{R}_{K K}-\frac{\boldsymbol{R}_{K N} \boldsymbol{R}_{K N}^{T}}{R_{N N}}\right) \mathbf{a}_{K}\right] .}
\end{aligned}
$$

From the Gaussian assumption, we have $q_{N} \sim \mathcal{N}\left(m_{q_{N}}, \sigma_{q_{N}}^{2}\right)$, where $m_{q_{N}}=\left(m_{N \mid K}+\frac{\boldsymbol{R}_{N K} \mathbf{a}_{K}}{R_{N N}}\right) / \sqrt{C_{N \mid K}^{a}}$ and $\sigma_{q_{N}}^{2}=1$. Therefore, it can be concluded that $q_{N}^{2}$ has a non-central chisquared distribution as $q_{N}^{2} \sim \chi^{2}(1, \lambda)$, with degree of freedom $k=1$ and non-centrality parameter

$$
\lambda=m_{q_{N}}=\left(m_{N \mid K}+\frac{\boldsymbol{R}_{N K} \mathbf{a}_{K}}{R_{N N}}\right) / \sqrt{C_{N \mid K}^{a}} .
$$

Using expression (26) and (16), we can give an expression for the robust placement criterion

$$
J_{P}\left(\mathbf{X}_{N}, \theta \mid \mathbf{a}_{K}\right)=\operatorname{Pr}\left(W_{N \mid K} \geqslant \theta\right)=1-Q_{\frac{1}{2}}\left(\sqrt{\lambda}, \sqrt{\theta^{\prime}}\right),
$$

where

$$
\theta^{\prime}=\frac{\theta-\mathbf{a}_{K}^{T}\left(\boldsymbol{R}_{K K}-\frac{\boldsymbol{R}_{K N} \boldsymbol{R}_{K N}^{T}}{R_{N N}}\right) \mathbf{a}_{k}}{R_{N N} C_{N \mid K}^{a}} .
$$

\section{OTHER SENSOR PLACEMENT CRITERIA}

This section contains a brief description of kriging based alternative criteria for sensor placement presented in the literature. We begin the section with a description of classical kriging based criteria for interpolation, more precisely, MI and entropy based criteria. Then, we present a criterion specially tailored for signal extraction, that is based on the expected predicted output SNR. We finish the section with a discussion on the advantages and disadvantages of each approach.

\section{A. Classical kriging criteria}

In the classical kriging approaches for sensor placement, the objective is to find sensor locations such that the set of measurements collected by the sensors provides the best estimation of the spatial gain $\hat{\mathbf{a}}(\mathbf{X})$ in the whole space $\mathcal{X}$. In this regard, several criteria have been proposed. Among them, entropy and MI are two basic tools to define an effective cost function. In what follows, we explain how these two measures can be used as criteria for sensor placement.

1) Entropy: Assume that $\mathcal{S}$ is the set of possible positions in the space for sensor placement. Based on the concept of entropy, the most informative subset $\mathcal{A} \subset \mathcal{S}$ with the size $|\mathcal{A}|=M$ to place $M$ sensors, is the one that maximizes the entropy, which implies that the spatial gain at the selected positions are mostly uncertain about each other. Therefore, the following problem needs to be solved [23]:

$$
\mathcal{A}=\underset{\mathcal{A} \subset \mathcal{S} ;|\mathcal{A}|=M}{\operatorname{argmax}} J_{H}\left(\boldsymbol{X}_{\mathcal{A}}\right),
$$

where $J_{H}\left(\boldsymbol{X}_{\mathcal{A}}\right)=H\left(\mathbf{a}_{\mathcal{A}}\right)$ is the entropy of the spatial gain at the set of positions $\mathcal{A}$. The entropy is defined as follows:

$$
H\left(\mathbf{a}_{\mathcal{A}}\right)=-\int_{-\infty}^{+\infty} p\left(\mathbf{a}_{\mathcal{A}}\right) \log p\left(\mathbf{a}_{\mathcal{A}}\right) d \mathbf{a}_{\mathcal{A}}
$$

where $\mathbf{a}_{\mathcal{A}}=\mathbf{a}\left(\boldsymbol{X}_{\mathcal{A}}\right)$ is the vector of spatial gain at positions $\boldsymbol{x}_{i} \in \mathcal{A}, i=\{1,2, \ldots, M\}$ and $p(\cdot)$ is its pdf.

According to [24], solving (30) is NP-hard. Therefore, a greedy approach can be used to find a near optimal solution to this problem [25], [26]. In the greedy approach, we assume 
that $K$ sensors have already been placed at position $\mathcal{A}_{K}=$ $\left\{\boldsymbol{x}_{1}, \boldsymbol{x}_{2}, \ldots, \boldsymbol{x}_{K}\right\}$, and we add a single sensor $(N=1)$ leading to a total number of sensors of $M=K+1$. Taking into account the Gaussian assumption on the model of the spatial gain as in (7), the conditional entropy is presented as follows:

$$
H\left(a_{N} \mid \mathbf{a}_{K}\right)=\frac{1}{2} \log C_{N \mid K}^{a}+\frac{1}{2}(\log (2 \pi)+1),
$$

where, $C_{N \mid K}^{a}$ is defined in (23). This criterion measures the uncertainty between the spatial gain at the new position $a_{N}$ and the spatial gain at the previously selected positions $\mathbf{a}_{K}$, and it is preferred to choose the location that maximizes this quantity. Therefore, by defining $J_{H}\left(\mathbf{x}_{N} \mid \mathbf{a}_{K}\right) \triangleq H\left(a_{N} \mid \mathbf{a}_{K}\right)$, the next sensor position given by this criterion is the following:

$$
\hat{\mathbf{x}}_{N}=\underset{\mathbf{x}_{N} \in \mathcal{S} \backslash \mathcal{A}_{K}}{\operatorname{argmax}} J_{H}\left(\mathbf{x}_{N} \mid \boldsymbol{a}_{K}\right) .
$$

The disadvantage of the above criterion is that the decision for sensor placement does not depend on the measurements collected by the sensors, and it only depends on the prior knowledge on the covariance function of the model of the spatial gain, i.e. $C^{a}$. Also, it is shown that the criterion attempts to push the sensors far from each other and scatters them in the boundaries of the space which causes the information to be lost and not be able to have a better estimation of the physical phenomenon [27]. This is due to the fact that the criterion just takes into account the entropy of the selected positions and it does not observe the entire space of the interest.

2) Mutual information (MI): Another way to tackle the problem of optimal sensor placement which resolves the weak point of the entropy is to use mutual information. In [16], it is suggested to maximize $M I\left(\boldsymbol{a}_{\mathcal{A}}, \boldsymbol{a}_{\mathcal{S} \backslash \mathcal{A}}\right)$ which is the mutual information between the selected locations for sensor placement $\mathcal{A}$ and the rest of the space $\mathcal{S} \backslash \mathcal{A}$ :

$$
\mathcal{A}=\underset{\mathcal{A} \subset \mathcal{S} ;|\mathcal{A}|=M}{\operatorname{argmax}} J_{M I}\left(\boldsymbol{X}_{\mathcal{A}}\right),
$$

where $J_{M I}\left(\boldsymbol{X}_{\mathcal{A}}\right)=H\left(\boldsymbol{a}_{\mathcal{S} \backslash \mathcal{A}}\right)-H\left(\boldsymbol{a}_{\mathcal{S} \backslash \mathcal{A}} \mid \boldsymbol{a}_{\mathcal{A}}\right)$. In [16], it is shown that this criterion outperforms the entropy criterion in (30). It is also proved that solving (34) is NP-hard, and therefore, a greedy algorithm is suggested to obtain an approximate solution. In this regard, it is proposed to add the sensors one by one, such that the increase of the mutual information is maximized. So, assuming that $K$ sensors have already been located at $\boldsymbol{X}_{K}$, and the new single sensor is supposed to be located at $\mathbf{x}_{N}$ in such a way that $M I\left(\mathbf{a}_{K+N}\right)-M I\left(\boldsymbol{a}_{K}\right)$ is maximized, then we have the following:

$$
\hat{\mathbf{x}}_{N}=\underset{\mathbf{x}_{N} \in \mathcal{S} \backslash \mathcal{A}_{K}}{\operatorname{argmax}} J_{M I}\left(\mathbf{x}_{N} \mid \boldsymbol{a}_{K}\right),
$$

where, $J_{M I}\left(\mathbf{x}_{N} \mid \boldsymbol{a}_{K}\right) \triangleq H\left(a_{N} \mid \mathbf{a}_{K}\right)-H\left(a_{N} \mid \mathbf{a}_{\mathcal{S} \backslash \mathcal{L}}\right)$, and $\mathcal{L}=$ $\left\{\boldsymbol{X}_{K} \cup \mathbf{x}_{N}\right\}$. Under the Gaussian assumption for the spatial gain, the above criterion is simplified as follows:

$$
\mathbf{x}_{N}=\underset{\mathbf{x}_{N} \in \mathcal{S} \backslash \mathcal{A}_{K}}{\operatorname{argmax}} \frac{C_{N N}^{a}-C_{N K}^{a}\left(C_{K K}^{a}\right)^{-1} C_{K N}^{a}}{C_{N N}^{a}-C_{N \mathcal{L}}^{a}\left(C_{\mathcal{L} \mathcal{L}}^{a}\right)^{-1} C_{\mathcal{L} N}^{a}} .
$$

\section{B. Expected SNR}

An alternative solution to deal with the uncertainty on the spatial gain is to consider the expected SNR as a sensor placement criterion [19]:

$$
J_{E}\left(\mathbf{X}_{M}\right)=\mathbb{E}\left[W_{M}\right] .
$$

From Section II, we know that $W_{M}$ is a sum of $M$ independent random variables $\gamma_{i}$ distributed according to (12). Therefore, the criterion above is given by the sum of the means of $\Gamma_{i}$. A simpler way to derive the criterion is to directly evaluate the average of the quadratic form (8) using the GP prior on $\hat{\mathbf{a}}(x)$ :

$$
\begin{aligned}
J_{E}\left(\mathbf{X}_{M}\right) & =\mathbb{E}\left[\hat{\mathbf{a}}_{M}^{T} \mathbf{R}_{M M} \hat{\mathbf{a}}_{M}\right] \\
& =\mathbf{m}_{M}^{T} \mathbf{R}_{M M} \mathbf{m}_{M}+\operatorname{Tr}\left(\mathbf{R}_{M M} \mathbf{C}_{M M}^{a}\right) \\
& =\operatorname{Tr}\left[\mathbf{R}_{M M}\left(\mathbf{m}_{M} \mathbf{m}_{M}^{T}+\mathbf{C}_{M M}^{a}\right)\right] .
\end{aligned}
$$

Note that here both the greedy and sequential approaches can be used as well. By considering that $K$ sensors have already been located at positions $\mathbf{X}_{K}$, that gain information $\mathbf{z}_{K}$ is available and that we want to add $N$ new sensors at $\boldsymbol{X}_{N}$, the criterion from [19] can be written as follows:

$$
\begin{aligned}
J_{E}\left(\mathbf{X}_{N} \mid \mathbf{z}_{K}\right) & =\mathbb{E}\left[\hat{\mathbf{a}}_{M}^{T} \boldsymbol{R}_{M M} \hat{\mathbf{a}}_{M} \mid \mathbf{z}_{K}\right] \\
& =\operatorname{Tr}\left[\mathbf{R}_{M M}\left(\mathbf{m}_{M \mid K} \mathbf{m}_{M \mid K}^{T}+\mathbf{C}_{M \mid K}^{a}\right)\right],
\end{aligned}
$$

where $\mathbf{m}_{M \mid K}$ and $\mathbf{C}_{M \mid K}^{a}$ are, respectively, the conditional mean and covariance given by (18) and (19).

\section{Discussion on different criteria}

In [19] it is shown that maximizing $J_{E}$, i.e. the expectation of the SNR, outperforms the classical kriging approaches based on the entropy $J_{H}$ and the mutual information $J_{M I}$ criteria for source estimation. This is clearly due to the fact that the $J_{E}$ criterion targets the SNR instead of the spatial gain for sensor placement. If we inspect expressions (38) and (39) for the expected SNR criterion, a remarkable difference with respect to $J_{H}$ and $J_{M I}$ is the presence of the mean of the gain. This difference may make the optimal sensor positions generated with $J_{E}$ differ substantially from those generated with $J_{M I}$ or $J_{H}$. For example, when the uncertainty on the gain is small ( $\sigma_{a}$ is small), $\mathbf{C}_{M \mid K}^{a}$ can be neglected. In this case, to maximize (39), the sensors need to be placed far enough, so that $\boldsymbol{R}_{M M}$ is close to a diagonal matrix. At the same time the elements of $\mathbf{m}_{K}$ and $\mathbf{m}_{M \mid K}$ should have large absolute values, so that the optimal positions will be spread on $\mathcal{X}$ but will be biased to the positions where the gain is large in absolute value. In the same situation, criteria $J_{H}$ and $J_{M I}$ will simply spread the positions in $\mathcal{X}$ in such a way that the gains on the sensors will be maximally uncorrelated.

It is expected that $J_{P}$ (28) outperforms $J_{H}$ and $J_{M I}$ for signal extraction, since it targets directly the SNR. As a downside, $J_{P}$ is generally more complex to evaluate than other criteria, including $J_{E}$, since it requires numerical evaluation of the cdf of a quadratic form of a Gaussian vector. The only case for $J_{P}$ which is comparable in terms of complexity to the other methods is either when $M=1$ or $N=1$. In these cases, $J_{P}$ is given in closed-form. Another drawback of $J_{P}$ when compared to $J_{E}$ is that it relies on the Gaussian assumption 
on the gain. For $J_{E}$, the GP model assumption on $a(\mathbf{x})$ is not necessary, it is only required that $a(\mathbf{x})$ is a stochastic process with mean $m^{a}(\mathbf{x})$ and covariance $C^{a}\left(\mathbf{x}, \mathbf{x}^{\prime}\right)$.

Although in terms of computational complexity, $J_{E}$ is simpler than $J_{P}$, in terms of robustness against uncertainty it may be inferior. The uncertainty of the SNR is summarized into its mean and by increasing the uncertainty of the spatial gain, the robustness of this criterion is decreased. We can see this behavior by inspecting (38) and (39). When the uncertainty becomes remarkable, the term depending on $C_{M \mid K}^{a}$ will bias the chosen locations to have large variance on their gains, thus generating positions which may lead to an uncertain SNR.

\section{NUMERICAL RESUlts}

Experiments concerning the robustness and performance of the proposed criterion $J_{P}$ and of the criteria from the literature $J_{E}, J_{H}$ and $J_{M I}$ are presented in this section ${ }^{1}$. To this end, we first present the simulation setup in section V-A. Next, in sections V-B and V-C, we briefly introduce three different metrics that we use in order to evaluate the performances of the criteria in terms of the robustness against the uncertainty of the spatial gain. In section V-E we will show the effect of the free parameter $\theta$ on the proposed criterion. In this section, we explain how parameter $\theta$ helps to control the robustness, and the improvement of the output SNR. This will help us to understand how to set this parameter in the later experiments. Also, in section V-D we analyze the effect of the smoothness of the noise and the spatial gain on the robustness of $J_{P}$. Afterwards, we compare the performance of different criteria in terms of the output SNR for a 1D and 2D setting in section V-F. Finally, in section V-G, we will compare the performance of different criteria in terms of estimating the spatial gain at all positions.

\section{A. Simulation setup}

In the numerical experiments, we consider a one dimension grid $\mathbf{X}_{T}=\left[\mathbf{x}_{1}, \mathbf{x}_{2}, \ldots, \mathbf{x}_{T}\right]$ in the normalized range $\mathbf{x}_{i} \in$ $[0,1]$ where $i=1,2, \ldots, T$ for possible sensor locations. Note that to keep the consistency of the paper, in the experimental part we represent the scalars with bold lowercase letters. Depending on the smoothness of the signals, the size of the grid $T$ and the number of initial sensors $K$, as well as their positions $\mathbf{X}_{K}$ will be changed in different simulation parts. In all cases, we assume that $\mathbf{m}_{K}=\mathbf{a}_{K}+\mathbf{b}_{K}+\overline{\mathbf{u}}_{K}$, where $\mathbf{m}_{K}$ is the mean of the spatial gain which will be used as the estimation of the spatial gain, $\mathbf{a}_{K}$ is the true value of the spatial gain, $\mathbf{b}_{K}$ is a bias in the spatial gain, and $\overline{\mathbf{u}}_{K}$ is the uncertainty of the spatial gain. Note that, the sum of the uncertainty and the bias represent the error $\mathbf{v}_{K}$ in equation (17) i.e. $\mathbf{v}_{K}=\mathbf{b}_{K}+\overline{\mathbf{u}}_{K}$. We use GP models $\mathcal{G P}\left(m(\mathbf{x}), C\left(\mathbf{x}, \mathbf{x}^{\prime}\right)\right)$, with a square exponential covariance function $C\left(\mathbf{x}, \mathbf{x}^{\prime}\right)=\sigma^{2} \exp \left(-\left(\mathbf{x}-\mathbf{x}^{\prime}\right)^{2} /\left(2 \rho^{2}\right)\right)$ to produce the spatial gain $a(\mathbf{x})$, the noise $n(\mathbf{x})$, and the uncertainty $\bar{u}(\mathbf{x})$. Here, $\rho$ is a smoothness parameter where a small $\rho$ means fast

\footnotetext{
${ }^{1}$ All the simulations in this section are done in MATLAB-R2018b on operating system macOS version 10.14.3, with processor 3.2 GB Intel Core i5 and memory 8 GB $1600 \mathrm{MHz}$ DDR3.
}

spatial changes, while a large $\rho$ means smooth changes. Also, the bias $b(\mathbf{x})$ is generated using a scaled GP such that the ratio between the bias and the spatial gain at each position remains intact for each Monte-Carlo realization. Note that when the GP is scaled in this way, it is not a GP anymore. The subscripts $(.)_{a},(.)_{n},(.)_{b}$, and $(.)_{u}$ refer to the GP parameters of the spatial gain, the noise, the bias and the uncertainty of the spatial gain, respectively. The smoothness parameters $\rho_{a}$, $\rho_{n}, \rho_{b}$, and $\rho_{u}$, and the variances $\sigma_{a}, \sigma_{n}, \sigma_{b}$, and $\sigma_{u}$ will be changed in different parts of the numerical experiments. We set all the mean functions $m(\mathbf{x})$ to be equal to 0 . Note that, in practice, we do not have the actual spatial gain $a(\mathbf{x})$, and in our simulations, as an oracle, we generate randomly one realization from a GP with zero-mean and covariance parameters $\sigma_{a}$ and $\rho_{a}$.

We note that in order to implement the proposed criterion $J_{P}\left(\mathbf{X}_{N}, \theta \mid \hat{\mathbf{a}}_{K}\right)$, it is required to choose a relevant value for the parameter $\theta$. So, we suggest the following model:

$$
\theta=\widehat{S N R}(\hat{\mathbf{f}})^{(K)}+\delta\left(\widehat{S N R}(\hat{\mathbf{f}})^{(K+N)}-\widehat{S N R}(\hat{\mathbf{f}})^{(K)}\right) .
$$

In the above equation, by using (21), $\widehat{S N R}(\hat{\mathbf{f}})^{(K)}$ and $\widehat{S N R}(\hat{\mathbf{f}})^{(K+N)}$ are the mean of the SNR before and after adding the new $N$ sensors, respectively. Setting $\theta$ according to the above model helps us to use a meaningful value for this parameter, which is relative to the initial value of the SNR added by a coefficient of the increase in the SNR after adding new sensors. In the rest of the simulation part, we parameterize $J_{P}$ as a function of $\delta$ instead of $\theta$. For the proposed method, we use the sequential approach, and we follow the steps presented in Algorithm 1.

\section{B. Evaluation metrics}

In order to evaluate the performance of different criteria in terms of robustness, in this part, we present three metrics, failure region (FR), Failure[\%], and false positive rate (FPR) that will be used in the experimental results.

Fig. 2 is provided to present the notions of FR, Failure[\%], and FPR. In this figure, the size of the grid is 300, and $K=3$ sensors have already been located at the positions $\mathbf{X}_{K}=\{0.05,0.5,0.95\}$, which are marked by circles. Here, we considered an unbiased situation i.e. $b(\mathbf{x})=0$, the standard deviations are set to be $\sigma_{a}=0.15, \sigma_{u}=0.15, \sigma_{n}=0.5$, and the smoothness parameters are $\rho_{a}=0.2, \rho_{u}=0.2, \rho_{n}=0.1$. The standard deviation of the source signal is $\sigma_{s}=2$. We use $J_{P}(15)$ as the placement criterion setting $\delta=0.5$. Now, the aim is to find the best location for the $4^{\text {th }}$ sensor. In this figure, the tilde superscript indicates that the function is normalized with its maximum value to be equal to one, and the initial value equal to zero. The normalized variation of SNR, denoted $\triangle \widehat{S N R}(\hat{\mathbf{f}})$, is as follows:

$$
\triangle \widetilde{S N R}(\hat{\mathbf{f}})=\frac{S N R(\hat{\mathbf{f}})-S N R_{\text {init }}(\hat{\mathbf{f}})}{S N R_{\max }(\hat{\mathbf{f}})-S N R_{\text {init }}(\hat{\mathbf{f}})},
$$

where $S N R_{\text {init }}(\hat{\mathbf{f}})$ represents the initial value of the SNR before adding the new sensor, and $S N R_{\max }(\hat{\mathbf{f}})$ is the maximum value of the SNR after adding the new sensor. Note 


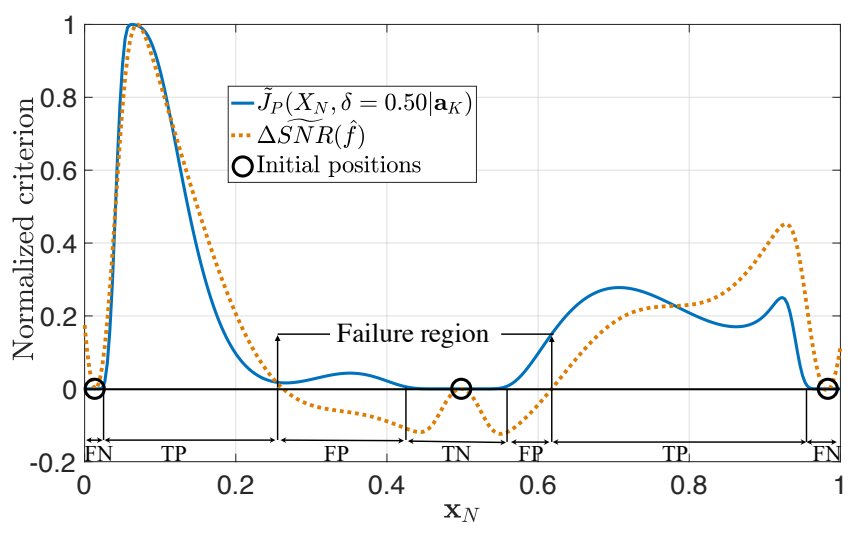

Fig. 2: Failure region is where the true value of the SNR with estimated extraction vector $\hat{\mathbf{f}}$ is smaller than its initial value before adding the new sensors. Here, the mean of the SNR is used as the criterion depicted with orange color. The blue curve represents the variation of the true SNR with estimated $\hat{\mathbf{f}}$. The tilde superscript represents the normalization of the criteria. Region classification: Positive $(P)$ vs. Negative $(N)$, and True $(T)$ vs. False $(F) . P$ and $N$ represent the regions where $\triangle \widehat{S N R}(\hat{\mathbf{f}})$ is positive and negative, respectively. $T$ is a situation when the criterion and $\Delta \overline{S N R}(\hat{\mathbf{f}})$ are both positive, or the criterion is zero and $\triangle \widehat{S N R}(\hat{\mathbf{f}})$ is negative. $F$ is when the criterion is positive while $\triangle \widehat{S N R}(\hat{\mathbf{f}})$ is negative, or the criterion is zero whenever $\widetilde{S N R}(\hat{\mathbf{f}})$ is positive.

that in this paper, the points of interest in the criteria are the locations of the maxima (and not their amplitudes), which are not affected by the normalization. Based on Fig. 2, the metrics FR, Failure[\%], and FPR will be discussed in the following.

Failure region (FR): Although $J_{E}$ and $J_{P}$ are criteria targeting SNR, uncertainty on $a(\mathbf{x})$ may lead to large values of these criteria at positions where in practice the value of the true output $\operatorname{SNR}\left(\hat{\mathbf{f}} \mid \mathbf{X}_{M}\right)$ may be decreased. The true SNR is given by (4), where $\mathbf{f}=\hat{\mathbf{f}}$ is the estimated extraction vector. This vector is given by $\hat{\mathbf{f}}=\mathbf{R}_{M M} \mathbf{m}_{M}$ in the non sequential approach and by $\hat{\mathbf{f}}=\mathbf{R}_{M M} \mathbf{m}_{M \mid K}$ in the sequential approach. As a consequence, the true output SNR can be rewritten as

$$
\operatorname{SNR}\left(\hat{\mathbf{f}} \mid \mathbf{X}_{M}\right)=\frac{\sigma_{s}^{2} \hat{\mathbf{f}}^{T} \mathbf{a}_{M} \mathbf{a}_{M}^{T} \hat{\mathbf{f}}}{\hat{\mathbf{f}}^{T} \mathbf{C}_{M M}^{n} \hat{\mathbf{f}}}
$$

Note that (42) differs from the estimated SNR, $\widehat{\mathrm{SNR}}(8)$, since this true SNR (42) depends on the true spatial gain $\mathbf{a}_{M}$, contrary to $\widehat{\mathrm{SNR}}$ which depends on the estimation of the spatial gain $\hat{\mathbf{a}}_{M}$. We call the set of positions that deteriorates the true $\mathrm{SNR}$ as failure region (FR).

In Fig. 2 it is seen that, due to the uncertainty, the normalized variation of the true SNR i.e. $\widehat{\triangle S N R}(\hat{\mathbf{f}})$ can take negative values at some regions, which means that by placing sensors at these positions, not only the SNR will not be improved, but it will even take smaller values compared to the initial SNR. Therefore, since $S N R(\hat{\mathbf{f}})<S N R_{\text {init }}(\hat{\mathbf{f}})$ in FR, the numerator of (41) becomes negative, and so, $\Delta \overline{S N R}(\hat{\mathbf{f}})$ becomes negative. In this figure, FR is marked with arrows. As depicted, although the true SNR takes quite smaller value in FR, the suggested criterion takes significant value at some positions in FR. In particular, the criterion has a local maxima at $\mathbf{x}=0.35$ which is inside FR, and so, a failure has happened due to the uncertainty. So, this is an example of the lack of robustness of this criterion.

Failure[\%]: To analyze the affect of the uncertainty and the bias in the model of the spatial gain on the size of the FR, we need to define a quantitative statistical measure. To do so, we can simulate $N_{M C}$ Monte-Carlo realizations of the gain, bias, uncertainty and noise GP, and then count the total number of positions within the FR, here denoted $N_{F R}$. For a total size of spatial grid $N_{T}$, we define the failure rate as follows:

$$
\text { Failure }[\%] \triangleq \frac{\text { Size of the FR }}{\text { Total size of the spatial grid }}=\frac{N_{F R}}{N_{T}} \text {. }
$$

The smaller the Failure[\%] is, the less uncertainty we have on the spatial gain.

FPR: In order to study the influence of the smoothness parameters $\rho_{a}$ and $\rho_{n}$ on the robustness of the criteria, we use the metric false positive rate (FPR). To do so, we first classify different regions of the space according to Fig. 2. According to this figure, we name the regions with positive $\widetilde{S N R}(\hat{\mathbf{f}})$ to be positive $(\mathrm{P})$, and the regions with negative $\widehat{\Delta S N R}(\hat{\mathbf{f}})$ to be negative regions $(\mathrm{N})$. Here, $\Delta \widetilde{S N R}(\hat{\mathbf{f}})$ denotes the normalized variation of the output SNR defined in (41). Accordingly, the true positive (TP) is the size of the region where both $\triangle \widehat{S N R}(\hat{\mathbf{f}})$ and the criterion are positive. The number of positions that $\widehat{\triangle S N R}(\hat{\mathbf{f}})$ is negative but the criterion takes a positive value is the so-called false positive (FP). The notation true negative (TN) corresponds to the size of the region where $\Delta \widehat{S N R}(\hat{\mathbf{f}})$ is negative, and the criterion is zero. Finally, false negative (FN) is related to the position with positive $\triangle \widehat{S N R}(\hat{\mathbf{f}})$, and zero value for the underlying criterion. Now, according to this region classification, and under different combinations of the values of $\rho_{a}$ and $\rho_{n}$, we calculate the false positive rate (FPR) of a criteria as follows:

$$
F P R=\frac{\text { false positive }}{\text { total number of negatives }}=\frac{F P}{T N+F P} .
$$

In above, the denominator is actually the size of the failure region (FR).

\section{Effect of uncertainty on the Failure [\%]}

In this part, we want to show how the effect of uncertainty and bias on Failure[\%]. To this end, Fig. 3 is provided. In this figure, three initial sensors are placed at $\mathbf{X}_{K}=$ $\{0.25,0.5,0.75\}$, and we place the $4^{t h}$ sensor using $J_{E}$. The parameters are set as $\rho_{a}=\rho_{u}=0.2, \rho_{n}=0.01, \sigma_{a}=0.5$ and $\sigma_{n}=0.01$. We generate $N_{M C}^{a}=10$ realizations for the spatial gain, and for each realization, we consider 50 runs for the bias, uncertainty and noise $\left(N_{M C}^{b}=N_{M C}^{u}=N_{M C}^{n}=50\right)$, 


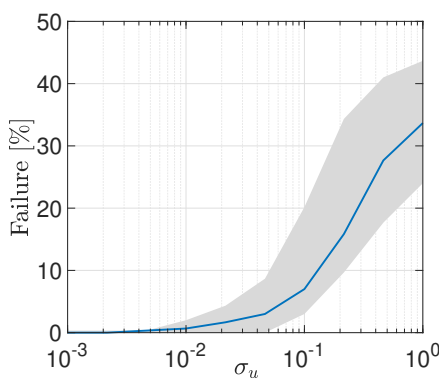

(a) The effect of uncertainty

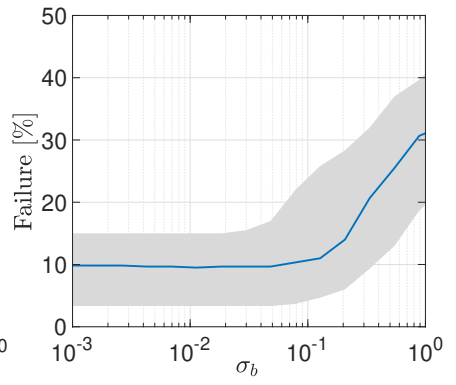

(b) The effect of bias
Fig. 3: Failure [\%] is a measure to compute the ratio of the sensor placement region which is in the failure region. (a) In this figure, the effect of the uncertainty level on the Failure $[\%]$ is depicted. Here, the bias is considered to be equal to 0 . (b) The effect of bias on Failure [\%] is depicted in this figure. The variance of the uncertainty is set equal to $\sigma_{u}=0.01$.

which leads to a total number of $N_{M C}=500$ Monte-Carlo (MC) realizations. Then, Failure [\%] is evaluated according to (43). In this figure, the effects of the bias and the uncertainty on Failure[\%] are studied separately. Firstly, in Fig. 3a, we consider an unbiased situation setting $b(\mathbf{x})=0$, and different uncertainty levels in the interval $\sigma_{u} \in\left[10^{-3} ; 10^{0}\right]$ are used. The blue curve is the average of Failure [\%] over all $N_{M C}$ realizations, and the gray shadow represents the standard deviation. As it was expected, by increasing $\sigma_{u}$, the average and the variance of Failure[\%] increases. This experiment is repeated in Fig. $3 b$ to study the effect of the bias. In this figure we use the previous configuration to set up the parameters, except that $\sigma_{u}$ is kept fixed to $\sigma_{u}=0.01$, and the level of the bias is changed in the range $\sigma_{b} \in\left[10^{-3} ; 10^{0}\right]$. As it can be seen, in average, the effect of the bias is negligible up to $\sigma_{b} \simeq 0.1$. If the level of bias goes beyond this value, the effect of the bias on Failure[\%] becomes significant, which means that the suggested model for the spatial gain is not appropriate, and it is required to apply better methods to provide a good approximation for the spatial gain. Since our proposed method is only focused on the uncertainty of the spatial gain, henceforth we assume that a suitable model is used for the spatial gain and we set $b(x)=0$.

Remark 7. It is noticeable that if the size of the FR is small, although the FPR gets large, the probability of selecting a position in FR is small. Conversely, if the size of the FR is large, although FPR gets small, the probability of selecting a sensor in FR can be large. To avoid these two marginal cases, according to Fig. 3, we set $\sigma_{u} \in[0.1,0.8]$ which provides a moderate Failure[\%] between $8 \%$ and $30 \%$.

\section{Effect of the smoothness of the spatial gain and noise correlation length-scale on robustness}

In this experiment, we want to show the effect of the smoothness of the spatial gain and the noise on the robustness. To this end, Fig. 4a is provided to show the robustness of the proposed criterion $J_{P}\left(\mathbf{x}_{N}, \delta \mid \mathbf{a}_{K}\right)$ in terms of the average $F P R[\%]$ and compares it with the criterion $J_{E}\left(\mathbf{x}_{N} \mid \mathbf{a}_{K}\right)$ for

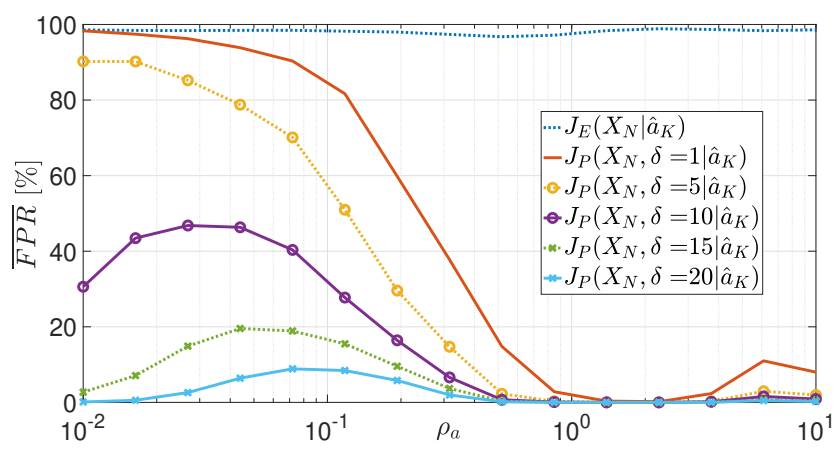

(a) The influence of a smoothness, with $\beta=0.1$.

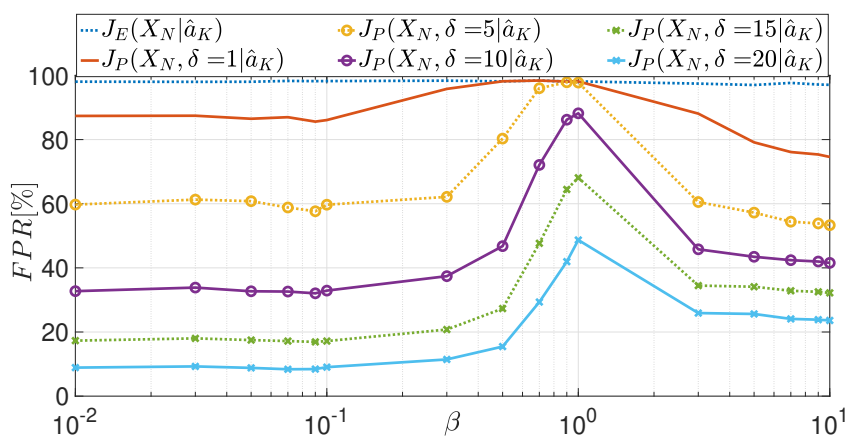

(b) The influence of smoothness ratio, with $\rho_{a}=0.1$.

Fig. 4: The effect of the smoothness of the spatial gain and the noise on the robustness. (a) Different smoothness values of the spatial gain are considered, and the smoothness ratio between the noise and the spatial gain is set as $\beta=\rho_{n} / \rho_{a}=0.1$. (b) Effect of the noise smoothness.

different smoothness conditions $\rho_{a}$. In this figure, three initial sensors are used at $X_{K}=\{0.05,0.5,0.95\}$ in a spatial grid of size 300 , and we look for the $4^{\text {th }}$ sensor position. The variances $\sigma_{s}=\sigma_{a}=\sigma_{u}=\sigma_{n}$ are all set to be equal to 1. The smoothness parameter $\rho_{a}$ takes 15 different values in the interval $\left[10^{-2}, 10\right]$ in the logarithmic scale, and for each case, we set $\rho_{u}=\rho_{a}$. The smoothness ratio between the spatial gain and the noise is fixed to be $\beta=\rho_{n} / \rho_{a}=0.1$. The total number of $N_{M C}=500$ realizations of the spatial gain, the mean, the uncertainty and the noise is considered. Note that the $x$-axis has a logarithmic scale in Fig. 4. $\overline{F P R}[\%]$ represents the average value of FPR[\%] over the whole Monte-Carlo realizations. As depicted in this figure, the proposed criterion is more robust compared to $J_{E}\left(\mathbf{x}_{N} \mid \hat{\mathbf{a}}_{K}\right)$. This figure shows that the larger the parameter $\delta$ is, the more robust the algorithm is against uncertainty for different $\rho_{a}$.

Now, the effect of the noise smoothness is shown in Fig. 4b. In this figure, we set $\rho_{a}=0.1$, and the values of the smoothness ratio $\beta=\rho_{n} / \rho_{a}$ are sampled in the interval $\left[10^{-2}, 10\right]$, thus leading to different values of $\rho_{n}$. As it is seen, again the proposed method shows a better performance as the smoothness of the noise is varied. Note that, for the proposed method, $\overline{F P R}[\%]$ takes its maximum value when the smoothness of the noise gets close to the smoothness of the spatial signal i.e. $\beta=\rho_{n} / \rho_{a}=1$. It is due to the fact that the smoothness similarity between the noise and the 


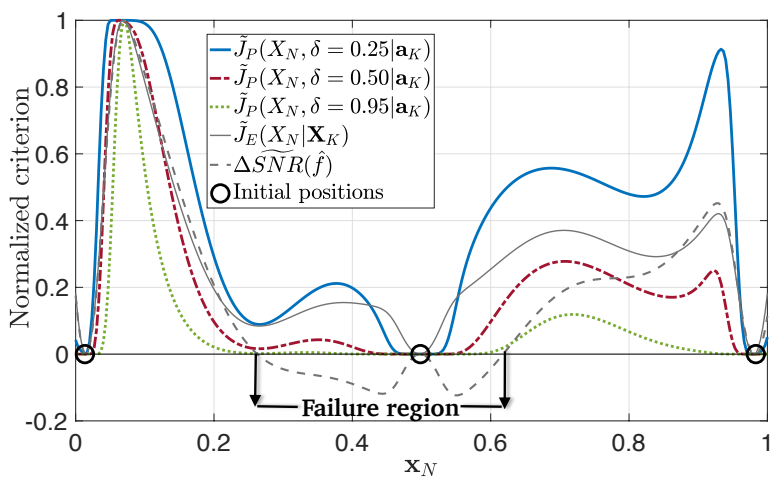

Fig. 5: Effect of parameter $\delta$. The solid curves are the proposed criterion for different $\delta$. The superscript tilde represents the normalization of the function. By increasing $\delta$, the algorithm is more robust against FR. However, other candidates for sensor placement which provide significant increase in the SNR can be ignored.

propagated source signal makes it difficult to separate them. It is also interesting to mention that for larger values of $\beta$, where the noise tends to be close to a constant function, the $\overline{F P R}[\%]$ starts to decrease. This result shows that whenever the noise is significantly different from the signal in terms of the smoothness, the $\overline{F P R}[\%]$ takes smaller values.

\section{E. Effect of $\delta$ and controlling the trade-off between robustness and average SNR maximization}

In this part, we study the effect of $\delta$ (which controls $\theta$ ) on the results obtained with $J_{P}$. To do so, Fig. 5 is provided. In this figure, the parameters are set as follows: $\rho_{a}=0.2, \rho_{u}=$ $0.2, \rho_{n}=0.1, \sigma_{a}=0.15, \sigma_{u}=0.15, \sigma_{n}=0.5$, and $\sigma_{s}=2$, with the size of the spatial grid equal to 300. Starting with three initial sensors at $\mathbf{X}_{K}=\{0.05,0.5,0.95\}$, we look for the best position for the $4^{\text {th }}$ sensor. The proposed criterion is used as the target function with three different values of $\delta(0.25,0.5$, and 0.95), which are depicted in Fig. 5 with a blue, red, and green color, respectively. Moreover, $\triangle \widetilde{S N R}(\hat{f})$ which is the output SNR normalized according to (41) is depicted with a dashed curve. The plots for $J_{E}\left(\mathbf{x}_{N} \mid \mathbf{X}_{K}\right)$ and $J_{P}\left(\mathbf{x}_{N}, \delta \mid \mathbf{X}_{K}\right)$ are normalized such that their maximum values be equal to 1 , and the initial values equal to 0 . The normalized forms are denoted by a superscript tilde. Note that the locations of maxima for the different criteria will not be changed by the normalization.

As depicted in Fig. 5, the proposed criterion behaves differently according to $\delta$. The larger this parameter is, the smaller the values of the criterion within FR are. It is noticeable that by increasing $\delta$, besides avoiding FR, it is probable to avoid some positions with significant increase in the SNR (e.g. for $\mathbf{x} \approx 0.95$ ). Consequently, high values of $\delta$ should be used to avoid locating the new sensor in FR, with the cost of achieving a smaller amount of increase in the SNR. Otherwise, we can decrease $\delta$ to keep most of the positions with a significant increase in the SNR, but this leads to an increased risk of having large values of the criterion for positions in FR. So, by choosing an appropriate $\delta$, we can make a trade-off between avoiding positions in the FR and keeping the regions with a high increase in the SNR.

We noted earlier that by increasing the $\delta$ parameter, the proposed method becomes more robust against the uncertainty of the spatial gain, and the FPR[\%] decreases. However, making the risk as small as possible, i.e. being too much strict against FR, may cause some good local maxima to be ignored, and consequently the algorithm becomes incapable to detect positions that provide a good output SNR. Therefore, it is important to make a trade-off between reducing the FPR[\%] and increasing the output SNR for choosing the parameter $\delta$. To this aim, in Fig. 6 we compare the behavior of the proposed method in terms of FPR and the improvement of the SNR under different uncertainty conditions and different selections of $\delta$. In this figure, we used a grid of size 100 between 0 and 1 for the sensor positions. An initial sensor is considered in the middle of the grid at $\mathbf{x}_{1}=0.5$, and we added 5 sensors using a greedy approach on the proposed criterion. The parameters are set as $\sigma_{a}=1, \rho_{a}=0.1, \sigma_{s}=1$, $\sigma_{n}=1$ and $\beta=0.2$. The variance of the uncertainty for the spatial gain varies as follows: $\sigma_{u} \in\{0,0.1,0.2, \ldots, 1\}$. We used $10 \mathrm{MC}$ realizations for $a(\mathbf{x})$ and $10 \mathrm{MC}$ realizations for $m_{a}(\mathbf{x})$. The average FPR denoted $\overline{F P R}$ is depicted as the blue curve with y-axis on the left, and the average improvement in the output SNR, i.e. $\overline{\Delta S N R(\hat{\mathbf{f}})}=\overline{S N R(\hat{\mathbf{f}})-S N R(\hat{\mathbf{f}})^{(i n i t)}}$ is presented in orange with the y-axis on the right. Here, $\operatorname{SNR}(\hat{\mathbf{f}})^{(i n i t)}$ denote the true value of the SNR at the initial situation with a unique sensor located at $\mathrm{x}=0.5$. Note that $S N R(\hat{\mathbf{f}})-S N R(\hat{\mathbf{f}})^{(i n i t)}$ is the variation of the SNR, which is equivalent to the numerator of (41). This experiment is repeated for three different values of $\delta$, namely 1,10 , and 20.

As Fig. 6 shows, by increasing the uncertainty $\sigma_{u}$, the FPR increases and the output improvement of SNR decreases. Furthermore, we can see that by choosing a small value for $\delta$, the output improvement of SNR takes larger value in average, however, the FPR becomes larger, meaning that the probability of being in the failure region increases. Comparing Fig. 6a with Fig. 6-b, we see that by increasing $\delta$ from 1 to 10, the average FPR becomes smaller for different values of $\sigma_{u}$. However, the average improvement in the SNR decreases. Going through a stricter selection for $\delta$, in Fig. 6-c we see that the average FPR gets quite smaller, providing a safe situation to avoid FR. Nevertheless, the improvement for the output SNR significantly decreases.

To conclude, we can say that, when the uncertainty is low, since the output SNR improvement can be large enough, it is possible to dedicate some SNR to reduce $\overline{F P R}[\%]$, and so, we can choose a larger $\delta$. In contrast, when $\sigma_{u}$ is large, since the output SNR is affected by this uncertainty, we cannot dedicate a large part of the SNR to have a smaller value of $\overline{F P R}[\%]$. Therefore, it is better not to be too strict, and select a moderate value for $\delta$. Depending on the application, we can make a trade-off between $\overline{\triangle S N R(\hat{\mathbf{f}})}$ and $\overline{F P R}[\%]$ by changing the parameter $\delta$ : in sensitive applications where decreasing the SNR is not acceptable, we need to choose a large $\delta$, even if the improvement of the output SNR is not large. On the other 


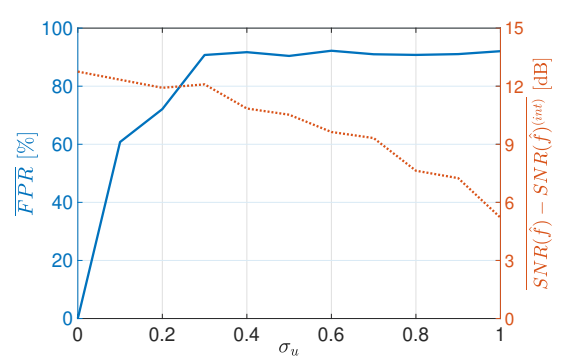

(a) $\delta=1$

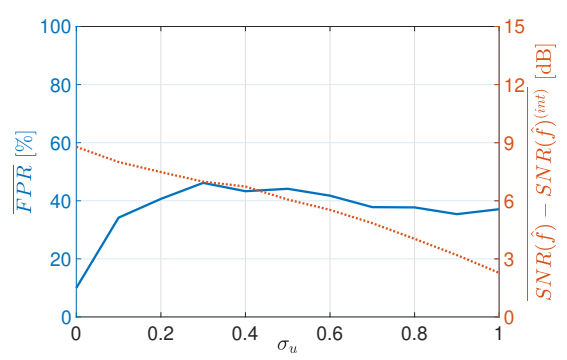

(b) $\delta=10$

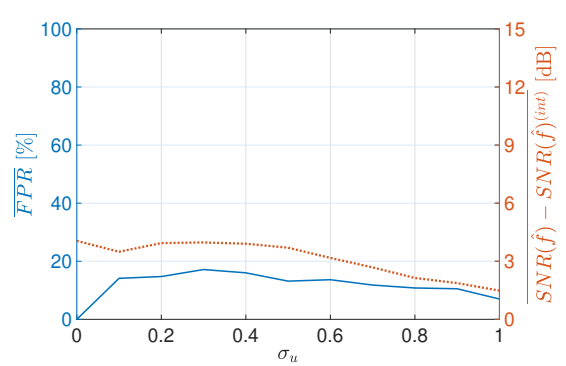

(c) $\delta=20$

Fig. 6: The trade-off between the robustness and the maximization of the averaged SNR improvement can be controlled by changing the parameter $\delta$. The blue curve with y-axis on the left represents the average $F P R[\%]$ according to different levels of uncertainty, and the orange curve with y-axis on the right depicts the average improvement of the SNR. From left to right, figures (a), (b), and (c) show the results for different values of $\delta$.

hand, in applications which are less sensitive to output SNR, we can take a risk of being trapped in the failure region to have a large improvement in the SNR, and, as a consequence, we can choose a smaller $\delta$.

\section{F. Sequential approach}

In this part, we compare the proposed method $J_{P}\left(\mathbf{x}_{N}, \delta \mid \mathbf{X}_{K}\right) \quad$ with $\quad J_{H}\left(\mathbf{x}_{N} \mid \mathbf{X}_{K}\right), \quad J_{M I}\left(\mathbf{x}_{N} \mid \mathbf{X}_{K}\right) \quad$ and $J_{E}\left(\mathbf{x}_{N} \mid \mathbf{X}_{K}\right)$ by using a sequential approach. To this end,

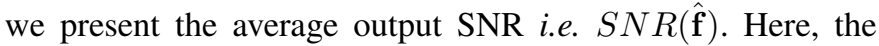
overline represents the average value of a measure over the total Monte-Carlo realizations. In the following, we first experiment with a one-dimensional setting. Later, we show results for a $2 \mathrm{D}$ case.

1) One-dimensional space: For setting up the experiments, we assume that $K=1$ initial sensor is located at $x_{1}=0.5$. Then we add sensors one by one up to $M=10$. Note that, since in this experiment we want to add up to 10 sensors to show the improvement of the algorithms according to the number of sensors, we chose the smoothness parameter of the spatial gain i.e. $\rho_{a}$ to be smaller than the previous experiments. In this way, we provide more variation for the spatial gain. Therefore, the source signal cannot be recovered just by using a very few sensors e.g. two or three sensors, and to recover the source signal with a desired accuracy, we need to collect more information by using sufficient number of sensors, which is up to 10 sensors in this experiment. Accordingly, the parameters are set as follows: $\rho_{a}=\rho_{u}=0.01, \beta=0.5, \sigma_{a}=\sigma_{n}=1$. We considered two different situations for $\sigma_{u}$. One is $\sigma_{u}=0.1$ which represents a small value for the level of uncertainty, and the other one is $\sigma_{u}=0.8$, which assumes a higher uncertainty. Depending on the level of uncertainty, we set $\delta=13$, and $\delta=10$ under the assumption of $\sigma_{u}=0.1$ and $\sigma_{u}=0.8$, respectively. Note that by reducing $\delta$ under higher uncertainty, we attempt to achieve a required minimum improvement in the average SNR, while having the same level of robustness measured by $\overline{F P R}[\%]$. In this part a number of $N_{M C}^{a}=10$ realizations are used for the spatial gain $\mathbf{a}(\mathbf{x})$, and for each realization we consider 10 runs for the bias, uncertainty and noise $\left(N_{M C}^{b}=N_{M C}^{u}=N_{M C}^{n}=10\right)$, leading to a total number of $N_{M C}=100$ Monte-Carlo realizations. For each realization of the spatial gain, we also have a new realization for the additive noise $\mathbf{n}(\mathbf{x})$.

Fig. 7 presents $\overline{\Delta S N R(\hat{\mathbf{f}})}$ by adding new sensors for two different uncertainty levels: a) $\sigma_{u}=0.1$, and b) $\sigma_{u}=0.8$. In this figure, the blue, red, orange, and green curves show the performances of the proposed criterion $J_{P}\left(\mathbf{x}_{N}, \delta \mid \hat{\mathbf{a}}_{K}\right)$, and other criteria based on the mean of the SNR $J_{E}\left(\mathbf{x}_{N} \mid \hat{\mathbf{a}}_{K}\right)$, the mutual information $J_{M I}\left(\mathbf{x}_{N} \mid \hat{\mathbf{a}}_{K}\right)$, and the entropy $J_{H}\left(\mathbf{x}_{N} \mid \hat{\mathbf{a}}_{K}\right)$, respectively.

We begin with a low level of uncertainty $\sigma_{u}=0.1$. By looking at Fig. 7-a, it is seen that the proposed criterion significantly outperforms the other methods and provides a larger value for $\triangle S N R(\hat{\mathbf{f}})$. By sequentially adding 10 sensors, $J_{P}\left(\mathbf{x}_{N}, \delta \mid \hat{\mathbf{a}}_{K}\right)$ provides $\Delta S N R(\hat{\mathbf{f}})=30 \mathrm{~dB}$, which still can be increased by adding more sensors. The next best criterion is $J_{E}\left(\mathbf{x}_{N} \mid \hat{\mathbf{a}}_{K}\right)$, which gives $\overline{\Delta S N R(\hat{\mathbf{f}})}=15 d B$. It is seen that unlike the proposed criterion, the other criteria cannot provide a better SNR by adding more sensors since their related curves have become almost flat. This is due to the uncertainty, which is not considered in these methods. It is important to mention that by adding a few number of sensors (up to 2-3 in this example), we do not see a significant difference in the performance of the proposed criterion and the one in $J_{E}\left(\mathbf{x}_{N} \mid \hat{\mathbf{a}}_{K}\right)$. It may be due to the fact that for a very few number of sensors, the information is not enough to have a good recovery of the source signal. However, immediately after adding the next sensors, due to the uncertainty, the proposed criterion starts outperforming $J_{E}\left(\mathbf{x}_{N} \mid \hat{\mathbf{a}}_{K}\right)$. Therefore, in this case, to avoid increasing the computational complexity, one can simply start adding primary sensors using $J_{E}\left(\mathbf{x}_{N} \mid \hat{\mathbf{a}}_{K}\right)$. Then, by increasing the number of sensors, it is recommended to use the proposed criterion as a robust method against uncertainty. It is interesting to mention that as claimed in [19], $J_{E}\left(\mathbf{x}_{N} \mid \hat{\mathbf{a}}_{K}\right)$ has notably a better performance compared to the classical kriging approaches $J_{H}\left(\mathbf{x}_{N} \mid \hat{\mathbf{a}}_{K}\right)$ and $J_{M I}\left(\mathbf{x}_{N} \mid \hat{\mathbf{a}}_{K}\right)$.

To continue, we analyze Fig. 7-b for $\sigma_{u}=0.8$. To compare the performance of the proposed method with the previous criteria, in Fig. 7-b, we see that the proposed criterion has a superior performance compared to the other methods in terms of $\overline{S N R}(\hat{\mathbf{f}})$. Fig. 7-b shows that by adding 10 sensors, the proposed criterion provides $\overline{S N R}(\hat{\mathbf{f}})=16 \mathrm{~dB}$, which 


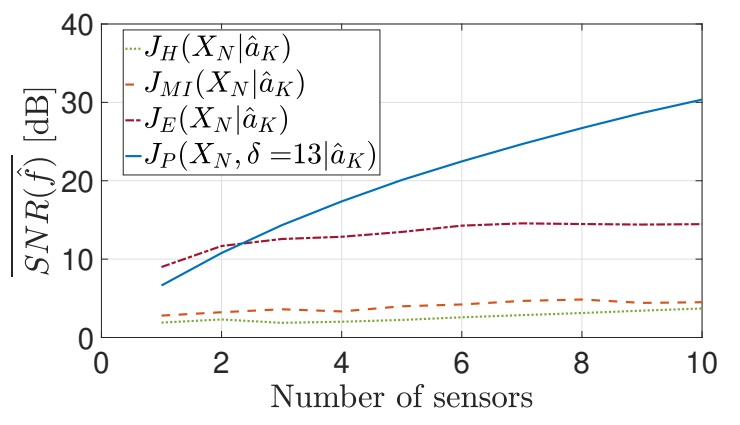

(a) $\sigma_{u}=0.1$

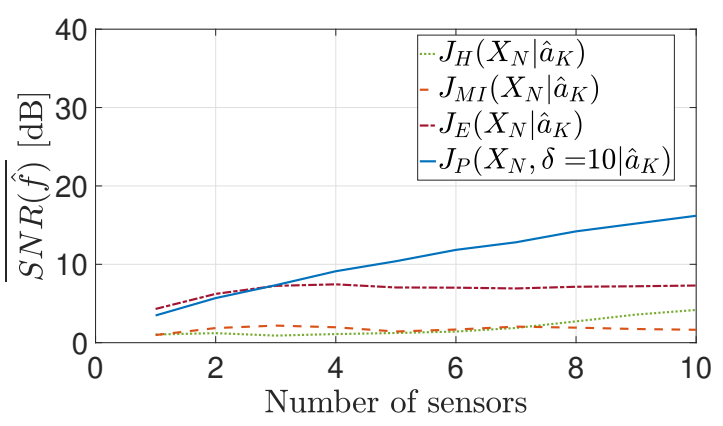

(b) $\sigma_{u}=0.8$

Fig. 7: In this figure, the performance of the proposed method is compared with the prior works by using a sequential approach. Here, the average output SNR is reported. Two situations are studied in this figure: a) $\sigma_{u}=0.1$, and b) $\sigma_{u}=0.8$.

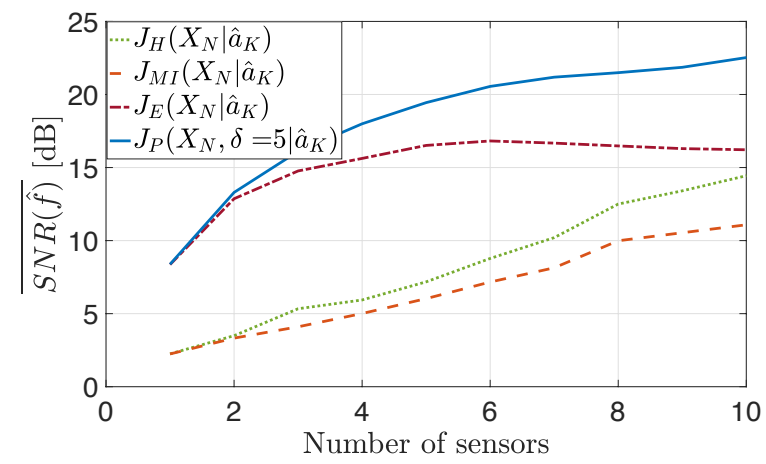

Fig. 8: In this figure, the performance of the proposed method in terms of the average output SNR is compared with the prior works by using a sequential approach. Here, we consider a 2D space, and we start with an initial sensor in the middle of the space at $(0.5,0.5)$. The standard deviation of the uncertainty on the spatial gain is set $\sigma_{u}=0.5$.

is $9 d B$ higher than the result achieved by $J_{E}\left(\mathbf{x}_{N} \mid \hat{\mathbf{a}}_{K}\right)$. Comparing Fig. 7-a and Fig. 7-b, it is seen that by increasing the uncertainty level from $\sigma_{u}=0.1$ to $\sigma_{u}=0.8$, the average output SNR decreases. For instance, considering that 10 sensors are added, for the proposed criterion, $\overline{S N R}(\hat{\mathbf{f}})$ drops from $30 d B$ in Fig. 7-a to $16 d B$ in Fig. 7-b. From the same point of view, the changes of the output SNR for $J_{E}\left(\mathbf{x}_{N} \mid \hat{\mathbf{a}}_{K}\right)$ is from $15 d B$ to $8 d B$, and for $J_{M I}\left(\mathbf{x}_{N} \mid \hat{\mathbf{a}}_{K}\right)$ it is from $4 d B$ to $2 d B$. The results for $J_{H}\left(\mathbf{x}_{N} \mid \hat{\mathbf{a}}_{K}\right)$ remain almost unchanged under different level of uncertainties.

2) Two-dimensional space : In this part we do experiments in a $2 \mathrm{D}$ setting. To this end, we consider a two-dimensional plane bounded between 0 and 1 in both dimensions. Also, we consider the size of the grid for sensor placement to be $20 \times 20$. Here, we consider that a single initial sensor (i.e. $K=1)$ is located in the middle of the space at $(0.5,0.5)$, and we add sensors one by one up to $M=10$. To have an appropriate variation for the spatial gain relative to the number of sensors, we set the smoothness parameter of the spatial gain $\rho_{a}=0.2$. Other parameters are set as follows: $\rho_{u}=\rho_{a} / 3, \beta=0.5, \sigma_{a}=1, \sigma_{u}=0.5$, and $\sigma_{n}=1.5$. In this part a number of 5 realizations are used for the spatial gain and the noise, and for each realization we consider 5 runs for the bias and the uncertainty $\left(N_{M C}^{b}=N_{M C}^{u}=5\right)$, leading to a total number of $N_{M C}=25$ Monte-Carlo realizations. Fig. 8 depicts the average output SNR i.e. $S N R(\hat{\mathbf{f}})$ by adding new sensors. As it is seen, from the very beginning when we have few sensors e.g. $M=2$, the proposed probabilistic criterion $J_{P}$ provides superior performance compared with the other criteria, and this superiority is increased by adding more sensors. For example, in the case of using $M=5$ sensors, we see that the proposed criterion $J_{P}$ provides $19.5 \mathrm{~dB}$ output $\mathrm{SNR}$, meanwhile, it is equal to $16.5 \mathrm{~dB}$ for the average SNR $J_{E}\left(\mathbf{x}_{N} \mid \hat{\mathbf{a}}_{K}\right)$, and about $7 \mathrm{~dB}$ and $6 \mathrm{~dB}$ for the entropy $J_{H}\left(\mathbf{x}_{N} \mid \hat{\mathbf{a}}_{K}\right)$ and mutual information $J_{M I}\left(\mathbf{x}_{N} \mid \hat{\mathbf{a}}_{K}\right)$, respectively. It is also worth mentioning that in this experiment, the average run time for the proposed criterion is $0.15 \mathrm{sec}$ using sequential approach, and $0.14 \mathrm{sec}$ without updating the estimation of the spatial gain. We can see that, the additional step in the sequential approach (updating the estimation of the spatial gain) has a negligible effect on the run-time. Also, the run-time is $0.02,1.2$, and $0.03 \mathrm{sec}$ for the entropy, mutual information, and the average SNR, respectively.

Fig. 9 is provided to show the positions of the 10 sensors selected by different criteria. In this figure, we have chosen one of the realizations of the spatial gain and the noise, and the field of the spatial gain $a(\mathbf{x})$ is depicted using contour plot. The circles show the sensor positions and the numerical index besides each circle indicates the order of sensors' positions. From this figure, we can see that the classical kriging approaches (figures 9-a and 9-b) put the sensors in regularly spaced positions. Meanwhile, we can see that the average SNR and the probabilistic criteria (figures 9-c and 9-d) choose the positions close to the maximizers of the magnitude of the spatial gain.

\section{G. Estimation of the spatial gain}

In this part, we want to investigate whether the criteria that result in the best estimate of the source also provide the best estimate of the spatial gain, or not. To this end, in this experiment, we consider a $2 \mathrm{D}$ setting. The parameters are set the same as in section V-F2. We start with an initial sensor in the middle of the space, and we sequentially add 10 sensors. Each time we add a new sensor, we use (18) to estimate the spatial gain at the rest of the positions $\mathbf{X}_{N}$ being conditioned on the value of the spatial gain at already located sensors. 

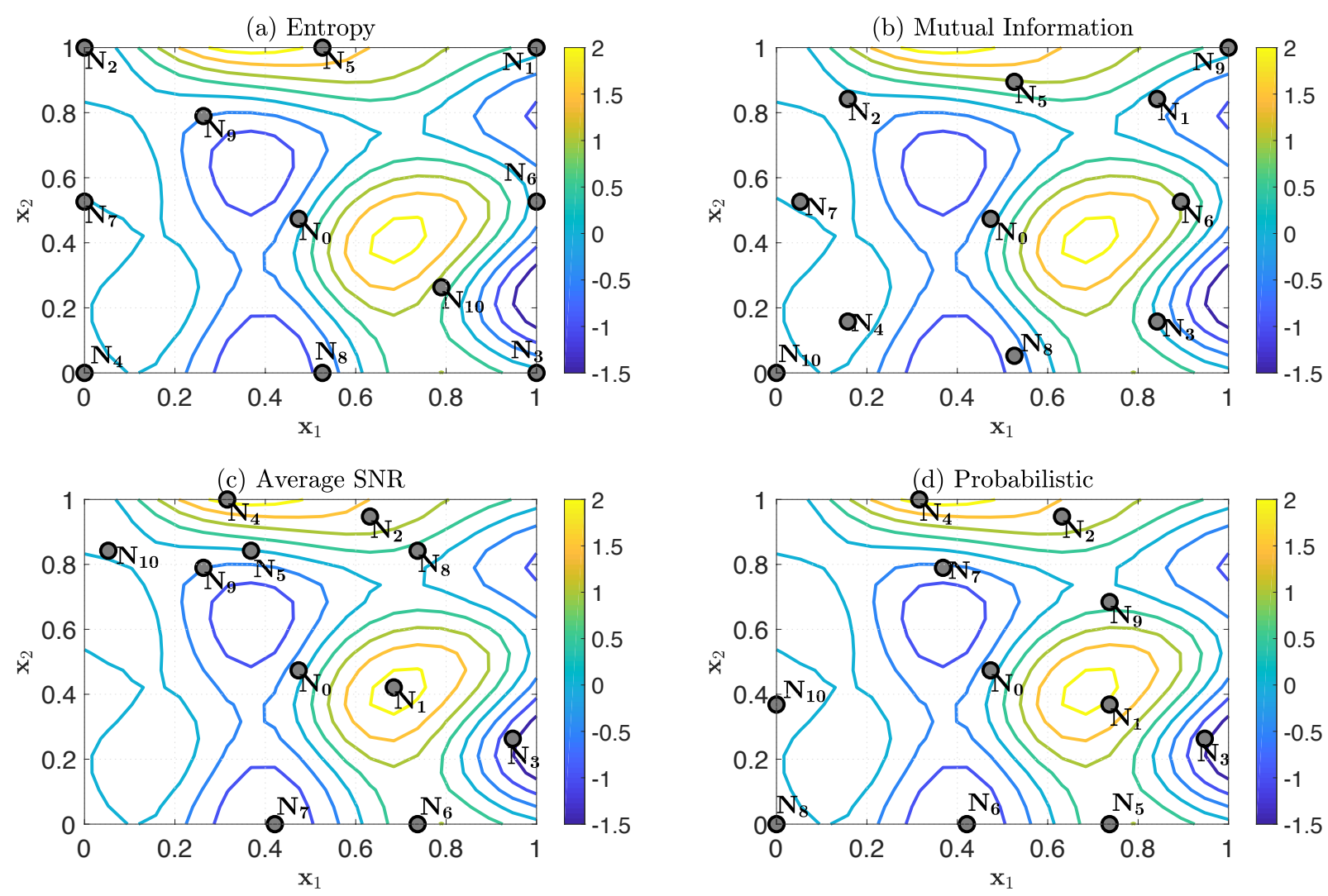

Fig. 9: This figure depicts the sensors' positions obtained from different criteria in a 2D space, by choosing one realization of the spatial gain and of the noise. The contour represents the true values of the spatial gain. We note that based on Fig. 8 the final output SNR for $100 \mathrm{MC}$ realizations is reported 11, 14, 16, and 23 (in dB) respectively for $J_{M I}, J_{H}, J_{E}$, and $J_{P}$.

Then, the mean square error (MSE) is calculated as follows:

$$
\operatorname{MSE}=\frac{1}{T} \sum_{i=1}^{T}\left(a\left(\mathbf{x}_{i}\right)-\hat{a}\left(\mathbf{x}_{i}\right)\right)^{2},
$$

where $T$ is the total size of the grid. The average MSE over 100 Monte-Carlo realizations for the spatial gain and the noise is presented in Fig. 10. We can see that the MSE is smaller using the classical kriging methods i.e. $J_{M I}$ and $J_{H}$, and the sensor positions obtained from the classical approach provide a better estimation (smaller MSE) of the spatial gain. Therefore, the criteria leading to the best estimation of the source, do not necessarily provide the best estimation of the spatial gain, and vice-versa.

\section{CONCLUSIONS AND PERSPECTIVES}

In this paper, we studied the problem of optimal sensor placement for signal extraction. We considered a linear signal extraction and targeted the predicted output SNR for sensor placement problem. Since the SNR is a continuous random variable in our setting, we presented a robust criterion for sensor placement based on the maximization of its cdf at a target SNR value.

Depending on the chosen target SNR value in the proposed criterion, we can make a trade-off between an improvement of the SNR and the robustness of the criterion. We also showed that the proposed criterion is derived from the distribution of

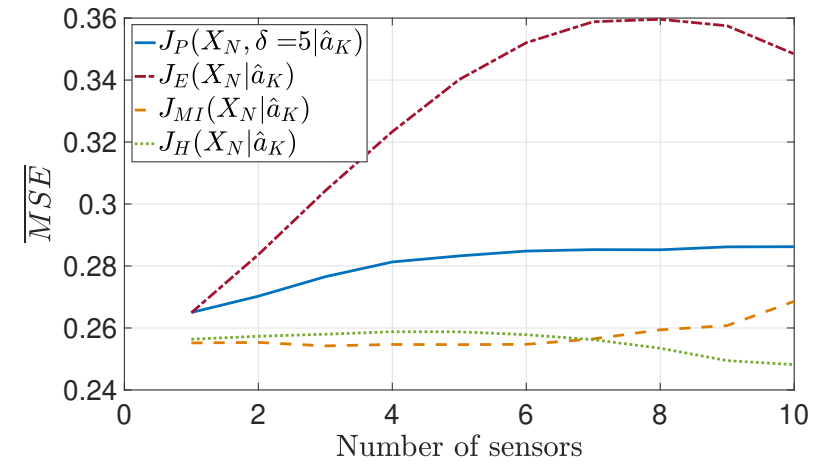

Fig. 10: Estimation of the spatial gain. In this figure, the performance of the proposed method in terms of the estimating the spatial gain is compared with the prior works by using a sequential approach.

the SNR so that the previously proposed criterion [19] can be seen as a special case of the present study. To reduce the computational cost of evaluating the criterion, we proposed a sequential approach where new sensor locations are chosen in batches. We presented how to update this sequential version when some information on the gains of the already placed batches of sensors is available. Numerical results showed a consistent superiority of the proposed criterion compared with classical kriging and the average SNR criteria in terms of 
the output SNR and robustness against the uncertainty on the model of spatial gain.

There are several future research topics regarding the proposed approach. First, we did not take into account the uncertainty on the hyperparameters of the models of the noise and spatial gain. This will be an important problem to be tackled in further studies. Second, in the sequential approach we have stated that information on the gains of the already placed sensors can be retrieved with ICA or SCA. In future work, such an approach where sensor placement is coupled with blind source separation techniques should be put into test. In this case, considering the case of multiple sources of interest (and not only one) would be interesting. Next, we saw that by considering higher dimensional settings e.g. 2D and 3D, searching in a grid will be prohibitively complex. Therefore, it will be necessary to develop a fast global optimization algorithm for maximizing our criterion. As an example, recently, a gradient-based technique is proposed in [28] that can be extended for the new criterion we proposed in this paper. Also, our study was focused on a linear instantaneous model to represent the sensor measurements. In future work, an extension of this approach to a more general convolutive mixture model can be considered.

In sensor selection problems, usually the guarantees that are given to greedy algorithm rely on the supermodularity property of the selection criterion [9], [10], [16]. In the future, it would be interesting to present such kind of guarantees for our placement criterion.

Finally, we mention that, although by adding new sensors we probably have improvement in $\overline{S N R}(\hat{\mathbf{f}})$, at the same time the computational complexity of the algorithm will be increased. Therefore, it is necessary to make a trade-off between increasing the SNR and decreasing the computational complexity to set the number of sensors. Using the Akaike information criterion (AIC) [29] to provide such a balance, we can propose a criterion which contains one term representing the improvement of the SNR and another term to measure the complexity of the algorithm.

\section{APPENDIX A \\ PROOF OF LEMMA-1}

Let vector $\mathbf{w}$ be a normally distributed random vector with zero mean and an identity covariance matrix $\mathbf{I}_{M}: \mathbf{w} \sim$ $\mathcal{N}\left(0, \mathbf{I}_{M}\right)$. Now, we define a random variable $y_{i}$ as follows:

$$
y_{i} \triangleq \mathbf{u}_{i}^{T}\left(\mathbf{w}+\mathbf{m}_{M}^{\prime}\right)
$$

where

$$
\mathbf{m}_{M}^{\prime}=\left(\boldsymbol{C}_{M M}^{a}\right)^{-\frac{1}{2}} \mathbf{m}_{M}^{a} .
$$

Note that this definition of the random variable $y_{i}$ provides an independence between $d_{i} y_{i}^{2}$ and $d_{j} y_{j}^{2}$ for $i \neq j$ which is studied in Remark 2.

Therefore, the squared form of $y_{i}$ becomes as follows:

$$
\begin{aligned}
y_{i}^{2} & =\left(\mathbf{w}+\mathbf{m}_{M}^{\prime}\right)^{T} \mathbf{u}_{i} \mathbf{u}_{i}^{T}\left(\mathbf{w}+\mathbf{m}_{M}^{\prime}\right) \\
& =\mathbf{w}^{T} \mathbf{u}_{i} \mathbf{u}_{i}^{T} \mathbf{w}+2\left(\mathbf{m}_{M}^{\prime}\right)^{T} \mathbf{u}_{i} \mathbf{u}_{i}^{T} \mathbf{w}+\left(\mathbf{m}_{M}^{\prime}\right)^{T} \mathbf{u}_{i} \mathbf{u}_{i}^{T}\left(\mathbf{m}_{M}^{\prime}\right) .
\end{aligned}
$$

According to the above equation, we have the following:

$$
\begin{aligned}
& \sum_{i=1}^{M} d_{i} y_{i}^{2}=\mathbf{w}^{T}\left[\sum_{i=1}^{M}\left(d_{i} \mathbf{u}_{i} \mathbf{u}_{i}^{T}\right)\right] \mathbf{w} \\
& +2\left(\mathbf{m}_{M}^{\prime}\right)^{T}\left[\sum_{i=1}^{M}\left(d_{i} \mathbf{u}_{i} \mathbf{u}_{i}^{T}\right)\right] \mathbf{w}+\left(\mathbf{m}_{M}^{\prime}\right)^{T}\left[\sum_{i=1}^{M}\left(d_{i} \mathbf{u}_{i} \mathbf{u}_{i}^{T}\right)\right] \mathbf{m}_{M}^{\prime} .
\end{aligned}
$$

Since $d_{i}$ 's and $\mathbf{u}_{i}$ 's are the eigenvalues and eigenvectors of $\boldsymbol{A}$, it yields $\sum_{i=1}^{M}\left(d_{i} \mathbf{u}_{i} \mathbf{u}_{i}^{T}\right)=\boldsymbol{A}$. So, we have

$$
\sum_{i=1}^{M} d_{i} y_{i}^{2}=\mathbf{w}^{T} \boldsymbol{A} \mathbf{w}+2\left(\mathbf{m}_{M}^{\prime}\right)^{T} \boldsymbol{A} \mathbf{w}+\left(\mathbf{m}_{M}^{\prime}\right)^{T} \boldsymbol{A}\left(\mathbf{m}_{M}^{\prime}\right)
$$

By replacing (47) in (48), and having $\boldsymbol{A} \triangleq$ $\left(\boldsymbol{C}_{M M}^{a}\right)^{\frac{1}{2}} \boldsymbol{R}_{M M}\left(\boldsymbol{C}_{M M}^{a}\right)^{\frac{1}{2}}$ from (10), we obtain the following: $\sum_{i=1}^{M} d_{i} y_{i}^{2}=\left(\mathbf{m}_{M}^{a}+\left(\boldsymbol{C}_{M M}^{a}\right)^{\frac{1}{2}} \mathbf{w}\right)^{T} \boldsymbol{R}_{M M}\left(\mathbf{m}_{M}^{a}+\left(\boldsymbol{C}_{M M}^{a}\right)^{\frac{1}{2}} \mathbf{w}\right)$.

Since $\mathbf{w}$ is a normally distributed random vector with zero mean and an identity covariance matrix, and also $\mathbf{m}_{M}^{a}$ and $C_{M M}^{a}$ are the mean vector and covariance matrix of the model of the spatial gain respectively, we conclude that $\mathbf{m}_{M}^{a}+\left(\boldsymbol{C}_{M M}^{a}\right)^{\frac{1}{2}} \mathbf{w}$ is equal to $\mathbf{a}_{M}$. So, we have the following:

$$
\sum_{i=1}^{M} d_{i} y_{i}^{2}=\mathbf{a}_{M}^{T} \boldsymbol{R}_{M M} \mathbf{a}_{M}^{T}=\left(1 / \sigma_{s}^{2}\right) \operatorname{SNR}\left(\mathbf{f}^{*} \mid \mathbf{X}_{M}\right)=W_{M} .
$$

\section{REFERENCES}

[1] J. W. Berry, L. Fleischer, W. E. Hart, C. A. Phillips, and J. P. Watson, "Sensor placement in municipal water networks," Journal of Water Resources Planning and Management, vol. 131, no. 3, May 2005.

[2] A. Krause, J. Leskovec, C. Guestrin, J. VanBriesen, and C. Faloutsos, "Efficient sensor placement optimization for securing large water distribution networks," Journal of Water Resources Planning and Management, vol. 134, no. 6, November 2008.

[3] C. K. Daniel, "Sensor placement for on-orbit modal identification and correlation of large space structures," Journal of Guidance, Control, and Dynamics, vol. 14, no. 2, pp. 251-259, 1991.

[4] M. Meo and G. Zumpano, "On the optimal sensor placement techniques for a bridge structure," Engineering Structures, vol. 27, no. 10, pp. 1488-1497, August 2005.

[5] S. Y. Chen and Y. F. Li, "Automatic sensor placement for model-based robot vision," IEEE Transactions on Systems, Man, and Cybernetics, Part B (Cybernetics), vol. 34, no. 1, pp. 393-408, February 2004.

[6] H. J. Hermens, B. Frenriks, C. Disselhorst-Klugb, and G. Rau, "Development of recommendations for SEMG sensors and sensor placement procedures," Journal of Electromyography and Kinesiology, vol. 10, no. 5, pp. 361-374, October 2000.

[7] A. L. D. Siqueira Junior, A. F. Spirandeli, R. Moraes, and V. Zarzoso, "Respiratory waveform estimation from multiple accelerometers: An optimal sensor number and placement analysis," IEEE journal of biomedical and health informatics, 2018.

[8] Summers. T, H, Cortesi. F, L, and Lygeros. J, "On submodularity and controllability in complex dynamical networks," IEEE Transactions on Control of Network Systems, vol. 3, no. 1, pp. 91 - 101, 2016.

[9] Zhang. H, Ayoub. R, and Sundaram. S, "Sensor selection for kalman filtering of linear dynamical systems: Complexity, limitations and greedy algorithms," Automatica, vol. 78, pp. 202-210, 2017.

[10] Tzoumas. V, Atanasov. N, A, Jadbabaie. A, and Pappas. G, J, "Scheduling nonlinear sensors for stochastic process estimation," American Control Conference (ACC), 2017.

[11] C. E. Rasmussen and C. K. I. Williams, Gaussian processes for machine learning, vol. 1, MIT press Cambridge, 2006. 
[12] D. G. Krige, "A statistical approach to some basic mine valuation problems on the witwatersrand," Journal of the Southern African Institute of Mining and Metallurgy, vol. 52, no. 6, pp. 119-139, 1951.

[13] N. Cressie, "The origins of kriging," Mathematical Geology, vol. 22, no. 3, pp. 239-252, 1990.

[14] T. J. Santner, B. J. Williams, and W. I. Notz, The design and analysis of computer experiments, Springer Science \& Business Media, 2013.

[15] D. R. Jones, M. Schonlau, and W. J. Welch, "Efficient global optimization of expensive black-box functions," Journal of Global optimization, vol. 13, no. 4, pp. 455-492, 1998.

[16] A. Krause, A. Singh, and C. Guestrin, "Near-optimal sensor placements in gaussian processes: Theory, efficient algorithms and empirical studies," Journal of Machine Learning Research, vol. 9, pp. 235-284, 2008.

[17] M. C. Shewry and H. P. Wynn, "Maximum entropy sampling," Journal of applied statistics, vol. 14, no. 2, pp. 165-170, 1987.

[18] J. Sacks, W. J. Welch, T. J. Mitchell, and H. P. Wynn, "Design and analysis of computer experiments," Statistical science, pp. 409-423, 1989.

[19] F. Ghayem, B. Rivet, C. Jutten, and R. Cabral Farias, "Optimal sensor placement for signal extraction," IEEE International Conference on Acoustics, Speech, and Signal Processing, pp. 4978-4982, May 2019.

[20] A. Nuttall and H. Albert, "Some integrals involving the q-m function," Transactions on Information Theory, vol. 21, no. 1, pp. 95 - 96, 1975.

[21] P. Comon, "Independent component analysis, a new concept?," Signal processing, vol. 36, no. 3, pp. 287-314, 1994.

[22] M. Zibulevsky and B. A. Pearlmutter, "Blind source separation by sparse decomposition in a signal dictionary," Neural computation, vol. 13, no. 4, pp. 863-882, 2001

[23] C. Currin, T. Mitchell, M. Morris, and D. Ylvisaker, "Bayesian prediction of deterministic functions, with applications to the design and analysis of computer experiments," Journal of the American Statistical Association, vol. 86, no. 416, pp. 953-963, 1991.

[24] C. Ko, J. Lee, and M. Queyranne, "An exact algorithm for maximum entropy sampling," Operations Research, vol. 43, no. 4, pp. 684-691, 1995.

[25] M. D. McKay, R. J. Beckman, and W. J. Conover, "A comparison of three methods for selecting values of input variables in the analysis of output from a computer code," Technometrics, vol. 21, no. 2, pp. 239$245,1979$.

[26] N. A. C. Cressie, "Statistics for spatial data," 1991.

[27] N. Ramakrishnan, C. Bailey-Kellogg, S. Tadepalli, and V.N. Pandey, "Gaussian processes for active data mining of spatial aggregates," Proceedings of the 2005 SIAM International Conference on Data Mining, p. 12,2005 .

[28] F. Ghayem, B. Rivet, C. Jutten, and R. Cabral, "Gradient-based algorithm with spatial regularization for optimal sensor placement," International Conference on Acoustics, Speech, and Signal Processing (ICASSP), 2020.

[29] H. Akaike, "Information theory and an extension of the maximum likelihood principle," Selected Papers of Hirotugu Akaike, , no. 1, pp. 199-213, 1998.

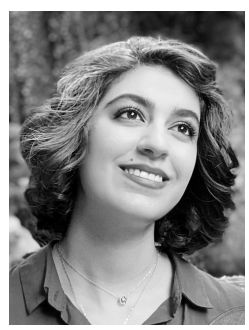

Fateme Ghayem received her B.Sc. degree in electrical engineering (communications), from the EE department, Shiraz University, Shiraz, Iran (2013). She also received her M.Sc. degree in biomedical engineering from the EE department, Sharif University of Technology, Tehran, Iran (2015). After finishing her M.Sc. study, she spent two years as a research assistant at DSP-lab, Sharif University of Technology, which was in collaboration with GIPSA-Lab, Grenoble, France. In 2020, Fateme received her Ph.D. degree at GIPSA-lab, University Grenoble Alpes, Grenoble, France. Her research is mainly focused on statistical signal processing, optimization, machine learning, source extraction, graph signal processing, and data fusion.

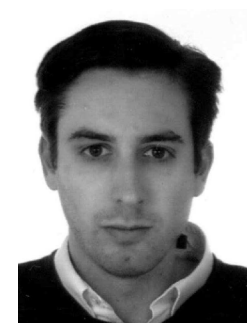

Bertrand Rivet was graduated from the École Normale Supérieure de Cachan, France. He received the Agrégation de Physique Appliquée in 2002, the Master's degree from the University of Paris-XI, France, in 2003 and the $\mathrm{PhD}$ degree from Grenoble Institute of Technology (GIT), France, in 2006.

Since 2007, he is an Associate Professor in signal processing with PHELMA and a member of GIPSAlab, GIT, France. His research concerns linear and non-linear source separation/extraction and sensors network based on multimodal recordings applied on biomedical signal processing, audiovisual speech processing and chemical data.

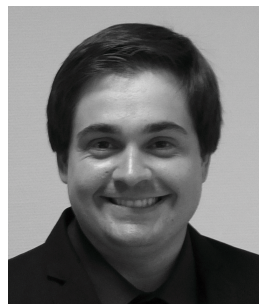

Rodrigo Cabral Farias was born in Porto Alegre, Brazil, in 1986. He received the B.Sc. degree in electrical engineering from the Federal University of Rio Grande do Sul (UFRGS), Porto Alegre, Brazil, and from the Grenoble Institute of Technology (Grenoble-INP), Grenoble, France, both in 2009. He received the M.Sc and Ph.D. degree in signal processing from Grenoble-INP and Grenoble University in 2009 and 2013 respectively. He worked as a post doctoral researcher from 2013 to 2016 at the Grenoble Laboratory of Image, Speech, Signal, and Automation (GIPSA-lab). Since 2016, he is a lecturer at the University Cote d'Azur in the Sophia-Antipolis Laboratory of Informatics, Signals, Systems (I3S). His research concerns statistical signal processing, tensor methods for data analysis and design of experiments.

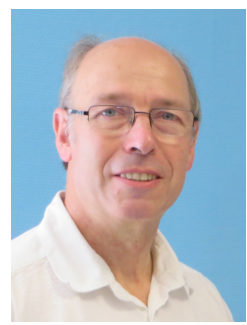

Christian Jutten (AM' 92-M' 03-SM' 06-F' 08) received Ph.D. (1981) and Doctor es Sciences (1987) degrees from Grenoble Institute of Technology, France. He was Associate Professor (1982-1989), Professor (1989-2019), and is Professor Emeritus since September 2019 at University Grenoble Alpes. Since 1980's, his research interests are in machine learning and source separation, including theory and applications (brain and hyperspectral imaging, chemical sensing, speech). He is author/co-author of four books, $120+$ papers in international journals and $250+$ publications in international conferences. He is a fellow of the IEEE and EURASIP.

He was a visiting professor at EPFL, RIKEN labs and University of Campinas. He served as the head of the signal/image processing laboratory, scientific advisor for signal/image processing at the French Ministry of Research (1996-1998) and CNRS (2003-2006 and 2012-2019).

$\mathrm{He}$ was organizer or program chair of many international conferences, including the first Independent Component Analysis Conference in 1999 (ICA'99) and IEEE MLSP 2009. He was the technical program co-chair of ICASSP 2020. He was a member of the IEEE MLSP and SPTM Technical Committees. He was associate editor in Signal Processing and IEEE Trans. on Circuits and Systems, and guest co-editor for IEEE Signal Processing Magazine (2014) and the Proceedings of the IEEE (2015). Since 2021, he is editor-in-chief of IEEE Signal Processing Magazine. 